\title{
On a Minimax Principle in Spectral Gaps
}

\author{
Albrecht Seelmann ${ }^{1}$
}

Received: 3 August 2021 / Accepted: 28 January 2022 / Published online: 3 March 2022

(c) The Author(s) 2022

\begin{abstract}
The minimax principle for eigenvalues in gaps of the essential spectrum in the form presented by Griesemer et al. (Doc Math 4:275-283, 1999) is adapted to cover certain abstract perturbative settings with bounded or unbounded perturbations, in particular ones that are off-diagonal with respect to the spectral gap under consideration. This in part builds upon and extends the considerations in the author's appendix to Nakić et al. (J Spectr Theory 10:843-885, 2020). Several monotonicity and continuity properties of eigenvalues in gaps of the essential spectrum are deduced, and the Stokes operator is revisited as an example.
\end{abstract}

Keywords Minimax values · Eigenvalues in gap of the essential spectrum · Block diagonalization $\cdot$ Stokes operator

Mathematics Subject Classification Primary 49Rxx; Secondary 47A10 • 47A75

\section{Introduction and Main Result}

The standard Courant minimax values $\lambda_{k}(A)$ of a lower semibounded operator $A$ on a Hilbert space $\mathcal{H}$ are given by

$$
\lambda_{k}(A)=\inf _{\substack{\mathfrak{M} \subset \operatorname{Dom}(A) \\ \operatorname{dim} \mathfrak{M}=k}} \sup _{\substack{x \in \mathfrak{M} \\\|x\|=1}}\langle x, A x\rangle=\inf _{\substack{\mathfrak{M} \subset \operatorname{Dom}\left(|A|^{1 / 2}\right) \\ \operatorname{dim} \mathfrak{M}=k}} \sup _{\substack{x \in \mathfrak{M} \\\|x\|=1}} \mathfrak{a}[x, x]
$$

Communicated by Jussi Behrndt.

This article is part of the topical collection "Spectral Theory and Operators in Mathematical Physics" edited by Jussi Behrndt, Fabrizio Colombo and Sergey Naboko.

$凶$ Albrecht Seelmann

albrecht.seelmann@math.tu-dortmund.de

1 Fakultät für Mathematik, Technische Universität Dortmund, 44221 Dortmund, Germany

Birkhäuser 
for $k \in \mathbb{N}$ with $k \leq \operatorname{dim} \mathcal{H}$, see, e.g., [23, Theorem 12.1] and also [31, Section 12.1 and Exercise 12.4.2]. Here, $\langle\cdot, \cdot\rangle$ denotes the inner product of $\mathcal{H}$, and $\mathfrak{a}$ with $\mathfrak{a}[x, x]=$ $\left\langle|A|^{1 / 2} x, \operatorname{sign}(A)|A|^{1 / 2} x\right\rangle$ for $x \in \operatorname{Dom}\left(|A|^{1 / 2}\right)$ is the form associated with $A$.

The above minimax values have proved to be a powerful description of the eigenvalues below the essential spectrum of $A$; in fact, they agree with these eigenvalues in nondecreasing order counting multiplicities as long as the latter exist and else equal the bottom of the essential spectrum. A standard application in this context is that the eigenvalues below the essential spectrum exhibit a monotonicity with respect to the operator: for two lower semibounded self-adjoint operators $A$ and $B$ with $A \leq B$ in the sense of quadratic forms one has $\lambda_{k}(A) \leq \lambda_{k}(B)$ for all $k$, see, e.g., [31, Corollary 12.3].

Matters get, however, much more complicated when eigenvalues in a gap of the essential spectrum are considered. If $A_{+}$is the (lower semibounded) part of $A$ associated with its spectrum in an interval of the form $(\gamma, \infty), \gamma \in \mathbb{R}$, then the minimax values for $A_{+}$still describe the eigenvalues of $A_{+}$below its essential spectrum, and thus the eigenvalues of $A$ in $(\gamma, \infty)$ below the essential spectrum of $A$ above $\gamma$. However, the subspaces over which the corresponding infimum is taken are chosen within the spectral subspace for $A$ associated with the interval $(\gamma, \infty)$ and therefore usually depend on the operator itself rather than just its domain. This makes it difficult to compare minimax values in spectral gaps of two different operators $A$ and $B$, even if their domains agree.

An adapted minimax principle taking this problem into account was first proposed by Talman [34] and Datta and Devaiah [2] in the context of Dirac operators. A corresponding mathematically rigorous result was announced by Esteban and Séré in [9] and proved together with Dolbeault in [5]. To the best of the authors knowledge, the first abstract theorem in this direction is due to Griesemer and Siedentop [12], the hypotheses of which have an overlap with $[5,9]$ but do not seem suitable to handle Dirac operators efficiently. In an attempt to overcome this and as a step towards finding the optimal assumptions, Griesemer, Lewis, and Siedentop [11] provided an alternative set of hypotheses. In a parallel development, Dolbeault, Esteban, and Séré [6] obtained an abstract theorem in an operator setting with yet another set of hypotheses that has an overlap with those of [11] but allows to deal with more potentials for Dirac operators. However, the abstract result of [11] does not seem to be contained in [6]. The result in [6] has later been extended to a form setting by Morozov and Müller [25], and recently by Schimmer, Solovej, and Tokus [28] to a class of symmetric operators with a distinguished self-adjoint extension. There has also been some activity regarding variational principles for block operator matrices, see, e.g., [19, 21] and triple variational principles, see, e.g., [7, 22]. They, however, follow a different approach and are not pursued here.

The aim of the present work is to complement the above works in an abstract framework. To this end, the result by Griesemer, Lewis, and Siedentop in [11] is adapted to a perturbative setting. In the particular case of bounded additive perturbations, this has already been done by the present author in the appendix to [27] with hypotheses that can, under reasonable assumptions, be verified explicitly by means of the DavisKahan $\sin 2 \Theta$ theorem from [3] or variants thereof. The latter has been successfully applied in [27] to study lower bounds on the movement of eigenvalues in gaps of 
the essential spectrum and of edges of the essential spectrum. In the current work, the considerations from [27, Appendix A] are extended and supplemented to cover also certain unbounded perturbations, in particular ones that are off-diagonal with respect to the spectral gap under consideration. The results obtained here seem not suitable to handle Dirac operators with Coulomb potentials since either the perturbation is assumed to be sufficiently small (Theorem 1.2) or of an off-diagonal structure (Theorem 1.4) or since they assume a semibounded setting (Theorems 1.3 and 1.5). However, weaker perturbations and other important situations such as perturbed periodic Schrödinger operators seem to be a natural context in which they can be applied. It should also be mentioned that some of the results and applications discussed here might at least in parts also be obtained with the approaches from earlier works such as $[6,22,25,28]$. This is commented on at various spots below, see, e.g., Remarks 1.6, 2.2(2), 2.11, and 2.13. The present work focuses on [11] as a starting point for mainly two reasons: Firstly, the proof of that result is remarkably elementary and short, while the proofs of $[6,22,25,28]$ are each a lot longer and much more technical, and, secondly, the techniques employed in Sects. 4 and 5 below to apply the approach from [11] promise to be of independent interest. In any case, to the best of the author's knowledge, neither the main results presented here nor their applications have been stated explicitly anywhere before. Rare exceptions to the latter are commented on accordingly.

\section{Main Results}

In order to formulate our main results, it is convenient to fix the following notational setup tailored towards spectral gaps to the right of 0; other gaps can of course always be reduced to this situation by spectral shift, cf. Remark 3.2 below.

Hypothesis 1.1 Let $A$ be a self-adjoint operator on a Hilbert space. Denote the spectral projections for $A$ associated with the intervals $(0, \infty)$ and $(-\infty, 0]$ by $P_{+}$and $P_{-}$, respectively, that is,

$$
P_{+}:=\mathrm{E}_{A}((0, \infty)), \quad P_{-}:=I-P_{+},
$$

and let

$$
\mathcal{D}_{ \pm}:=\operatorname{Ran} P_{ \pm} \cap \operatorname{Dom}(A), \quad \mathfrak{D}_{ \pm}:=\operatorname{Ran} P_{ \pm} \cap \operatorname{Dom}\left(|A|^{1 / 2}\right) .
$$

Moreover, let $B$ be another self-adjoint operator on the same Hilbert space with analogously defined spectral projections

$$
Q_{+}:=\mathrm{E}_{B}((0, \infty)), \quad Q_{-}:=I-Q_{+},
$$

and denote by $\mathfrak{b}$ the form associated with $B$, that is,

$$
\mathfrak{b}[x, y]=\left\langle|B|^{1 / 2} x, \operatorname{sign}(B)|B|^{1 / 2} y\right\rangle
$$

for $x, y \in \operatorname{Dom}[\mathfrak{b}]=\operatorname{Dom}\left(|B|^{1 / 2}\right)$. 
Here, $\mathrm{E}_{A}$ and $\mathrm{E}_{B}$ stand for the projection-valued spectral measures for the operators $A$ and $B$, respectively, and Ran $P_{ \pm}$denotes the range of $P_{ \pm}$. We have also used the notation $I$ for the identity operator.

Denoting the form associated with $A$ by $\mathfrak{a}$, the minimax values of the positive part $\left.A\right|_{\text {Ran } P_{+}}$of $A$ can clearly be written as

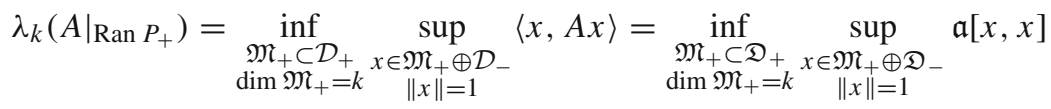

for $k \in \mathbb{N}$ with $k \leq \operatorname{dim} \operatorname{Ran} P_{+}$. The point of interest is now to find conditions on $B$ under which the minimax values for the positive part $\left.B\right|_{\operatorname{Ran} Q_{+}}$of $B$ admit the same representations with $\langle x, A x\rangle$ and $\mathfrak{a}[x, x]$ replaced by $\langle x, B x\rangle$ and $\mathfrak{b}[x, x]$, respectively, but with the infima taken over the same respective families of subspaces as for $A$ above. It is natural to consider this in a perturbative framework where $B$ is obtained by an operator or form perturbation of $A$ and, thus, one has $\operatorname{Dom}(A)=\operatorname{Dom}(B)$ and/or $\operatorname{Dom}\left(|A|^{1 / 2}\right)=\operatorname{Dom}\left(|B|^{1 / 2}\right)$.

In the situation of Hypothesis 1.1, a representation for the minimax values of $\left.B\right|_{\operatorname{Ran} Q_{+}}$of the above mentioned form is guaranteed by [25, Theorem 1] in the form setting with $\operatorname{Dom}\left(|A|^{1 / 2}\right)=\operatorname{Dom}\left(|B|^{1 / 2}\right)$ if

$$
\sup _{x_{-} \in \mathfrak{D}_{-}} \mathfrak{b}\left[x_{-}, x_{-}\right] \leq 0<\inf _{x_{+} \in \mathfrak{D}_{+} \backslash\{0\}_{x_{-}} \in \mathfrak{D}_{-}} \sup _{x_{-}} \frac{\mathfrak{b}\left[x_{+}+x_{-}, x_{+}+x_{-}\right]}{\left\|x_{+}+x_{-}\right\|^{2}}
$$

or by [6,Theorem 1.1] in the operator setting with $\operatorname{Dom}(A)=\operatorname{Dom}(B)$ if the analogous condition with $\mathfrak{D}_{ \pm}$replaced by $\mathcal{D}_{ \pm}$is satisfied; as pointed out in [28], for the latter additionally the restriction $\left.P_{-} B\right|_{\text {Ran }} P_{-}$should be essentially self-adjoint on $\mathcal{D}_{-}$, and it is very likely that a similar additional assumption is also necessary in the form setting of [25,Theorem 1], namely that the restriction of the form $\mathfrak{b}$ to $\mathfrak{D}_{-} \times \mathfrak{D}_{-}$is closable. Obviously, (1.1) and its operator analogue do not need any knowledge of $Q_{ \pm}$. Moreover, in case of (1.1), the right-hand side of (1.1) then agrees with $\lambda_{1}\left(\left.B\right|_{\operatorname{Ran} Q_{+}}\right)$, so that the strict inequality in (1.1) is also a necessary condition for such a representation to hold if $B$ has a spectral gap to the right of zero. On the other hand, this strict inequality is not always very convenient to verify or it is sometimes not even entirely clear how to verify it, cf. Remarks 1.6(2) and 2.2 (2) below. However, for an example where it can be verified analytically in terms of a Hardy-type inequality in the case of the Coulomb-Dirac operator, see [4]; cf. also [8] and [28,Section 3].

Instead of (1.1), [11] used the conditions

$$
\sup _{x_{-} \in \mathfrak{D}_{-}} \mathfrak{b}\left[x_{-}, x_{-}\right] \leq 0 \quad \text { and } \quad\left\|(|A|+I)^{1 / 2} P_{+} Q_{-}(|A|+I)^{-1 / 2}\right\|<1
$$

cf. Remark 3.3 below, which the authors were able to handle in case of Dirac operators but where especially the second condition seems to be hard to deal with in a general abstract setting. However, although (1.1) can treat more Coulomb-like potentials than (1.2) in case of the Dirac operator, (1.2) does not seem to imply (1.1) directly. 
In the main results below the aim is to discuss situations where the second condition in (1.2) can be replaced by $\left\|P_{+} Q_{-}\right\|<1,\left\|P_{+}-Q_{+}\right\|<1$, or by a certain explicit structural assumption on how $B$ is related to $A$. Here, especially the first two conditions seem to be natural since they relate the subspaces $\operatorname{Ran} P_{+}$and $\operatorname{Ran} Q_{+}$. Four results in this direction are presented here, each addressing a different situation, which are not contained in the previously known results in the sense that their hypotheses do not seem to imply (1.1), its operator analogue, or (1.2) directly. We first treat the case of operator perturbations and start with the direct extension of [27,Theorem A.2] to infinitesimal perturbations. Recall that an operator $V$ with $\operatorname{Dom}(V) \supset \operatorname{Dom}(A)$ is called $A$-bounded with $A$-bound $b_{*} \geq 0$ if for all $b>b_{*}$ there is some $a \geq 0$ with

$$
\|V x\| \leq a\|x\|+b\|A x\| \quad \text { for all } x \in \operatorname{Dom}(A)
$$

and if there is no such $a$ for $0<b<b_{*}$. If $b_{*}=0$, then $V$ is called infinitesimal with respect to $A$.

Theorem 1.2 Assume Hypothesis 1.1. Suppose, in addition, that $B$ is of the form $B=$ $A+V, \operatorname{Dom}(B)=\operatorname{Dom}(A)$, with some symmetric operator $V$ that is infinitesimal with respect to $A$. Furthermore, suppose that we have $\left\|P_{+} Q_{-}\right\|<1$ and that

$$
\langle x, B x\rangle \leq 0 \text { for all } x \in \mathcal{D}_{-} .
$$

Then,

$$
\lambda_{k}\left(\left.B\right|_{\text {Ran } Q_{+}}\right)=\inf _{\substack{\mathfrak{M}_{+} \subset \mathcal{D}_{+} \\ \operatorname{dim} \mathfrak{M}_{+}=k}} \sup _{\substack{x \in \mathfrak{M}_{+} \oplus \mathcal{D}_{-} \\\|x\|=1}}\langle x, B x\rangle=\inf _{\substack{\mathfrak{M}_{+} \subset \mathfrak{D}_{+} \\ \operatorname{dim} \mathfrak{M}_{+}=k}} \sup _{\substack{x \in \mathfrak{M}_{+} \oplus \mathfrak{D}_{-} \\\|x\|=1}} \mathfrak{b}[x, x]
$$

for all $k \in \mathbb{N}$ with $k \leq \operatorname{dim} \operatorname{Ran} P_{+}$.

It is worth to note that every operator of the form $B=A+V$ as in Theorem 1.2 is automatically self-adjoint on $\operatorname{Dom}(B)=\operatorname{Dom}(A)$ by the well-known Kato-Rellich theorem. Two more remarks regarding Theorem 1.2 are in order: (1) also certain perturbations $V$ that are not infinitesimal with respect to $A$ can be considered here, but at the cost of a stronger assumption on $\left\|P_{+} Q_{-}\right\|$, see Remark 4.2 below; (2) the condition $\left\|P_{+} Q_{-}\right\|<1$ is satisfied if the stronger inequality $\left\|P_{+}-Q_{+}\right\|<1$ holds. In the latter case, the subspaces $\operatorname{Ran} P_{+}$and $\operatorname{Ran} Q_{+}$automatically have the same dimension, that is, $\operatorname{dim} \operatorname{Ran} P_{+}=\operatorname{dim} \operatorname{Ran} Q_{+}$, see Remark 3.5 (a) below.

The stronger condition $\left\|P_{+}-Q_{+}\right\|<1$ just mentioned in fact also opens the way to employ a different approach than the one used to prove Theorem 1.2. This alternative approach has previously been used in the context of block diagonalization of operators and forms, see Sect. 5 below, and is particularly attractive if the unperturbed operator $A$ is semibounded.

Theorem 1.3 Assume Hypothesis 1.1. Suppose, in addition, that A is semibounded and that $\left\|P_{+}-Q_{+}\right\|<1$. 
If $\operatorname{Dom}\left(|A|^{1 / 2}\right)=\operatorname{Dom}\left(|B|^{1 / 2}\right)$ and $\mathfrak{b}[x, x] \leq 0$ for all $x \in \mathfrak{D}_{-}$, then

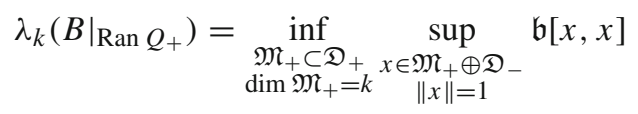

for all $k \leq \operatorname{dim} \operatorname{Ran} P_{+}=\operatorname{dim} \operatorname{Ran} Q_{+}$. If even $\operatorname{Dom}(A)=\operatorname{Dom}(B)$ and $\langle x, B x\rangle \leq 0$ for all $x \in \mathcal{D}_{-}$, then also

$$
\lambda_{k}\left(\left.B\right|_{\operatorname{Ran} Q_{+}}\right)=\inf _{\substack{\mathfrak{M}_{+} \subset \mathcal{D}_{+} \\ \operatorname{dim} \mathfrak{M}_{+}=k}} \sup _{\substack{x \in \mathfrak{M}_{+} \oplus \mathcal{D}_{-} \\\|x\|=1}}\langle x, B x\rangle
$$

for all $k \leq \operatorname{dim} \operatorname{Ran} P_{+}=\operatorname{dim} \operatorname{Ran} Q_{+}$.

It should be emphasized that the conditions $\operatorname{Dom}(A)=\operatorname{Dom}(B)$ and $\langle x, B x\rangle \leq 0$ for all $x \in \mathcal{D}_{-}$in Theorem 1.3 indeed imply that one has also $\operatorname{Dom}\left(|A|^{1 / 2}\right)=$ $\operatorname{Dom}\left(|B|^{1 / 2}\right)$ and $\mathfrak{b}[x, x] \leq 0$ for all $x \in \mathfrak{D}_{-}$, see Lemma 3.4 below. Note also that in contrast to Theorem 1.2, Theorem 1.3 makes no assumptions on how the operator $B$ is related to $A$. The latter will, however, be relevant when the hypotheses of Theorem 1.3 are to be verified in concrete situations.

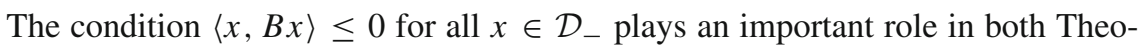
rems 1.2 and 1.3. In the case where $B=A+V$ with some $A$-bounded symmetric operator $V$, this condition is automatically satisfied if $\langle x, V x\rangle \leq 0$ for all $x \in \mathcal{D}_{-}$ since $\langle x, A x\rangle \leq 0$ holds for all $x \in \mathcal{D}_{-}$by definition. The latter is certainly the case for nonpositive $V$. Another instance of perturbations satisfying $\langle x, V x\rangle \leq 0$ for all $x \in \mathcal{D}_{-}$are so-called off-diagonal perturbations with respect to the decomposition Ran $P_{+} \oplus \operatorname{Ran} P_{-}$, in which case also the condition $\left\|P_{+}-Q_{+}\right\|<1$ can be verified efficiently. In comparison with Theorem 1.2, we may even relax the assumption on the $A$-bound of $V$ here.

Theorem 1.4 Assume Hypothesis 1.1. Suppose, in addition, that $B$ has the form $B=$ $A+V, \operatorname{Dom}(B)=\operatorname{Dom}(A)$, with some symmetric A-bounded operator $V$ with $A$-bound smaller than 1 and which is off-diagonal on $\operatorname{Dom}(A)$ with respect to the decomposition Ran $P_{+} \oplus \operatorname{Ran} P_{-}$, that is,

$$
P_{+} V P_{+} x=0=P_{-} V P_{-} x \quad \text { for all } x \in \operatorname{Dom}(A)
$$

Then, one has $\operatorname{dim} \operatorname{Ran} P_{+}=\operatorname{dim} \operatorname{Ran} Q_{+}$and

$$
\lambda_{k}\left(\left.B\right|_{\operatorname{Ran} Q_{+}}\right)=\inf _{\substack{\mathfrak{M}_{+} \subset \mathcal{D}_{+} \\ \operatorname{dim} \mathfrak{M}_{+}=k}} \sup _{\substack{x \in \mathfrak{M}_{+} \oplus \mathcal{D}_{-} \\\|x\|=1}}\langle x, B x\rangle=\inf _{\substack{\mathfrak{M}_{+} \subset \mathfrak{D}_{+} \\ \operatorname{dim} \mathfrak{M}_{+}=k}} \sup _{\substack{x \in \mathfrak{M}_{+} \oplus \mathfrak{D}_{-} \\\|x\|=1}} \mathfrak{b}[x, x]
$$

for all $k \in \mathbb{N}$ with $k \leq \operatorname{dim} \operatorname{Ran} Q_{+}$.

It is again worth to note that every operator of the form $B=A+V$ as in Theorem 1.4 is automatically self-adjoint on $\operatorname{Dom}(B)=\operatorname{Dom}(A)$ by the Kato-Rellich theorem. Moreover, although off-diagonal perturbations may seem a bit restrictive, 
they appear quite naturally when a general, not necessarily off-diagonal, perturbation is decomposed into its diagonal and off-diagonal parts. How Theorem 1.4 may then be applied is demonstrated in Proposition 2.12 below and the considerations thereafter.

The method of proof for Theorem 1.4 can to some extend be carried over to offdiagonal form perturbations, at least in the semibounded setting. The latter restriction is commented on in Sect. 5 below.

Theorem 1.5 Assume Hypothesis 1.1. Suppose, in addition, that B is semibounded and that its form $\mathfrak{b}$ is given by $\mathfrak{b}=\mathfrak{a}+\mathfrak{v}, \operatorname{Dom}[\mathfrak{b}]=\operatorname{Dom}[\mathfrak{a}]$, where $\mathfrak{a}$ is the form associated with $A$ and $\mathfrak{v}$ is a symmetric sesquilinear form satisfying

$$
\mathfrak{v}\left[P_{+} x, P_{+} y\right]=0=\mathfrak{v}\left[P_{-} x, P_{-} y\right] \quad \text { for all } x, y \in \operatorname{Dom}[\mathfrak{a}] \subset \operatorname{Dom}[\mathfrak{v}]
$$

and

$$
|\mathfrak{v}[x, x]| \leq a\|x\|^{2}+b|\mathfrak{a}[x, x]| \quad \text { for all } x \in \operatorname{Dom}\left(|A|^{1 / 2}\right)=\operatorname{Dom}[\mathfrak{a}]
$$

with some constants $a, b \geq 0$.

Then, one has $\operatorname{dim} \operatorname{Ran} P_{+}=\operatorname{dim} \operatorname{Ran} Q_{+}$and

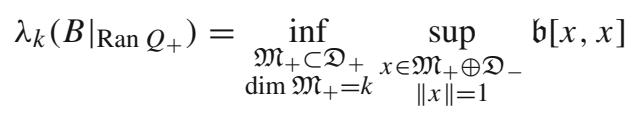

for all $k \in \mathbb{N}$ with $k \leq \operatorname{dim} \operatorname{Ran} Q_{+}$.

The semiboundedness of $B$ in Theorem 1.5 forces $A$ to be semibounded as well, see the proof of Theorem 1.5 below. In this regard, Theorem 1.5 can be interpreted as a particular case of the first part of Theorem 1.3 with $\operatorname{Dom}\left(|A|^{1 / 2}\right)=\operatorname{Dom}\left(|B|^{1 / 2}\right)$, in which the remaining hypotheses are automatically satisfied due to the structure of the perturbation.

Remark 1.6 (1) If $B$ in Theorem 1.5 is lower semibounded, then the operator $(|B|+I)^{1 / 2} Q_{-}$is everywhere defined and bounded, and so is the operator $(|B|+I)^{1 / 2} Q_{-} P_{+}$. Taking into account that $\mathfrak{b}[x, x]=\mathfrak{a}[x, x] \leq 0$ for $x \in \mathfrak{D}_{-}$ and $\mathfrak{b}[x, x]=\mathfrak{a}[x, x]>0$ for $x \in \mathfrak{D}_{+}$, Theorem 1.5 therefore reproduces in this situation a particular case of the earlier result [12, Theorem 3].

(2) If in Theorems 1.4 or 1.5 the unperturbed operator $A$ has a spectral gap to the right of 0 , then considerations as in part (1) show that (1.1) or the corresponding analogue in the operator framework is satisfied; cf. also Corollaries 2.5 and 2.7 (a) below. In this regard, Theorems 1.4 and 1.5 then can also be deduced from [6] and [25], respectively. However, if $A$ does not have such a gap, it is a priori not clear how to derive the two theorems from $[6,25]$.

The rest of this note is organized as follows. In Sect. 2 we discuss applications of the main theorems and revisit the Stokes operator as an example in the framework of Theorem 1.5. It is also explained there how the framework for off-diagonal perturbations in Theorem 1.4 can be applied to general, not necessarily off-diagonal, perturbations 
by decomposing the perturbation into its diagonal and off-diagonal parts. Section 3 is devoted to an abstract minimax principle based on [11]. Two approaches are then used to verify the hypotheses of this abstract minimax principle, the graph norm approach and the block diagonalization approach, respectively, which are discussed separately in Sects. 4 and 5 below. Theorem 1.2 is proved in Sect. 4, which is based on the author's appendix to [27] and extends the corresponding considerations to certain unbounded perturbations. Theorems 1.3-1.5 are proved in Sect. 5, which builds upon recent developments on block diagonalization of operators and forms from [24] and [14], respectively. Finally, Appendix A reproduces the proof from [11] for the abstract minimax principle discussed in Sect. 3, and Appendix B provides some consequences of the well-known Heinz inequality that are used at various spots in this work and are probably folklore.

\section{Applications and Examples}

In this section, we use the main results from Sect. 1 to prove monotonicity and continuity properties of minimax values in gaps of the essential spectrum in various situations and also revisit the well-known Stokes operator in the framework of Theorem 1.5 as an example. We finally discuss how to apply the off-diagonal framework from Theorem 1.4 to general, not necessarily off-diagonal, perturbations.

We first consider the situation of indefinite or semidefinite bounded perturbations, which has partially been discussed in a slightly different form in [27]. For a bounded self-adjoint operator $V$ we define bounded nonnegative operators $V^{(p)}$ and $V^{(n)}$ with $V=V^{(p)}-V^{(n)}$ via functional calculus by

$$
V^{(p)}:=(1+\operatorname{sign}(V)) V / 2, \quad V^{(n)}:=(\operatorname{sign}(V)-1) V / 2 .
$$

We clearly have $\left\|V^{(p)}\right\| \leq\|V\|$ and $\left\|V^{(n)}\right\| \leq\|V\|$.

The following result can be proved in several ways. The proof below is based on Theorem 1.2 and is in its core close to the proofs of Theorems 3.14 and 3.15 in [27]. An alternative proof for part (b) based on Theorem 1.4 is discussed after Remark 2.13 below. The result itself extends Theorem 5 in [12], which was formulated there for bounded nonpositive $V$ that are relatively compact with respect to the unperturbed operator $A$.

Proposition 2.1 Let the finite interval $(c, d)$ belong to the resolvent set of the selfadjoint operator $A$, and let $V$ be a bounded self-adjoint operator on the same Hilbert space satisfying $\left\|V^{(p)}\right\|+\left\|V^{(n)}\right\|<d-c$ with $V^{(p)}$ and $V^{(n)}$ as in (2.1). Set $P:=$ $E_{A}([d, \infty))$ and $Q:=E_{A+V}\left(\left[d-\left\|V^{(n)}\right\|, \infty\right)\right)$. Then:

(a) The interval $\left(c+\left\|V^{(p)}\right\|, d-\left\|V^{(n)}\right\|\right)$ belongs to the resolvent set of the operator $A+V$, and we have $\|P-Q\|<1$.

(b) With $\mathcal{D}_{+}:=\operatorname{Ran} P \cap \operatorname{Dom}(A)$ and $\mathcal{D}_{-}:=\operatorname{Ran}(I-P) \cap \operatorname{Dom}(A)$ we have

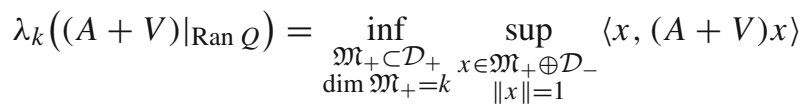


for all $k \in \mathbb{N}$ with $k \leq \operatorname{dim} \operatorname{Ran} Q$.

(c) With $\mathcal{E}_{+}:=\operatorname{Ran} Q \cap \operatorname{Dom}(A)$ and $\mathcal{E}_{-}:=\operatorname{Ran}(I-Q) \cap \operatorname{Dom}(A)$ we have

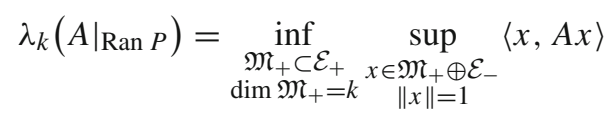

for all $k \in \mathbb{N}$ with $k \leq \operatorname{dim} \operatorname{Ran} P$.

Proof(a). This is Proposition 2.1 and Theorem 1.1 in [33], respectively; cf. also [36,Theorem 3.2]. More precisely, the variant of the Davis-Kahan $\sin 2 \Theta$ theorem in [33,Theorem 1.1] gives

$$
\|P-Q\| \leq \sin \left(\frac{1}{2} \arcsin \frac{\left\|V^{(p)}\right\|+\left\|V^{(n)}\right\|}{d-c}\right)<\frac{\sqrt{2}}{2}<1 .
$$

In particular, the subspaces $\operatorname{Ran} P$ and $\operatorname{Ran} Q$ have the same dimension; cf. also Remark 3.5 (1) below.

(b). Pick $\gamma \in\left(c+\left\|V^{(p)}\right\|, d-\left\|V^{(n)}\right\|\right)$. By part (a) we then have $\mathrm{E}_{A-\gamma}((0, \infty))=P$ and $\mathrm{E}_{A+V-\gamma}((0, \infty))=Q$. Moreover, for $x \in \mathcal{D}_{-}$we have

$$
\begin{aligned}
\langle x,(A+V-\gamma) x\rangle & =\langle x,(A-\gamma) x\rangle+\left\langle x, V^{(p)} x\right\rangle-\left\langle x, V^{(n)} x\right\rangle \\
& \leq\left(c-\gamma+\left\|V^{(p)}\right\|\right)\|x\|^{2}<0 .
\end{aligned}
$$

In light of part (a), the claim now follows from Theorem 1.2 with $P_{+}=P$ and $Q_{+}=Q$ upon a spectral shift by $\gamma$.

(c). Similarly as in (b), pick $\gamma \in\left(c+\left\|V^{(p)}\right\|+\left\|V^{(n)}\right\|, d\right)$. We then have $\mathrm{E}_{A-\gamma}((0, \infty))=P$ and for some chosen $\rho \in\left(c+\left\|V^{(p)}\right\|, d-\left\|V^{(n)}\right\|\right)$ also $\mathrm{E}_{A+V-\rho}((0, \infty))=Q$. Moreover, for $x \in \mathcal{E}_{-}$we have

$$
\begin{aligned}
\langle x,(A-\gamma) x\rangle & =\langle x,(A+V-\gamma) x\rangle-\left\langle x, V^{(p)} x\right\rangle+\left\langle x, V^{(n)} x\right\rangle \\
& \leq\left(c+\left\|V^{(p)}\right\|-\gamma+\left\|V^{(n)}\right\|\right)\|x\|^{2}<0 .
\end{aligned}
$$

In light of part (a), the claim now follows analogously from Theorem 1.2 with switched roles of $A$ and $A+V$ and with $P_{+}=Q$ and $Q_{+}=P$.

Remark 2.2 (1) A corresponding representation of the minimax values in terms of the forms associated with $A+V$ and $A$, respectively, as in Theorems 1.2-1.4 holds here as well. However, for the sake of simplicity and since this is not needed in Corollaries 2.3 and 2.4 below, this has not been formulated in Proposition 2.1.

(2) Part (b) of Proposition 2.1 can also be deduced from [6]. Indeed, for $y \in \mathcal{D}_{+}$we analogously have

$$
\langle y,(A+V-\gamma) y\rangle \geq\left(d-\gamma-\left\|V^{(n)}\right\|\right)\|y\|^{2}>0,
$$

which together with (2.2) implies that the operator analogue to condition (1.1) is satisfied. However, the situation is less clear for part (c) of Proposition 2.1. Here, 
for $y \in \mathcal{E}_{+}$we get

$$
\langle y,(A-\gamma) y\rangle \geq\left(d-\left\|V^{(n)}\right\|-\gamma-\left\|V^{(p)}\right\|\right)\|y\|^{2},
$$

which is positive only if $\gamma<d-\left\|V^{(p)}\right\|-\left\|V^{(n)}\right\|$. Together with the condition $\gamma>c+\left\|V^{(p)}\right\|+\left\|V^{(n)}\right\|$ in (2.3), this requires the stronger assumption $\left\|V^{(p)}\right\|+\left\|V^{(n)}\right\|<(d-c) / 2$. The latter can be somehow remedied with a continuity argument, but in the end the approach presented in the proof of Proposition 2.1 above is just more convenient.

The above proposition includes the particular cases where $V$ satisfies the condition $\|V\|<(d-c) / 2$ and where $V$ is semidefinite with $\|V\|<d-c$, which in the context of part (c) have essentially been discussed in the proofs of Theorems 3.14 and 3.15 in [27]. However, Proposition 2.1 allows also certain indefinite perturbations $V$ with $(d-c) / 2 \leq\|V\|<d-c$ that were not covered before and may thus be used to refine the results in [27].

As immediate corollaries to Proposition 2.1, we obtain the following monotonicity and continuity statements for the minimax values in gaps of the essential spectrum, which, in essence, reproduce particular cases of results in [36].

Corollary 2.3 Let $A$ be as in Proposition 2.1, and let $V_{0}$ and $V_{1}$ be bounded self-adjoint operators on the same Hilbert space satisfying the condition max

$$
\left\{\left\|V_{0}^{(p)}\right\|+\left\|V_{0}^{(n)}\right\|,\left\|V_{1}^{(p)}\right\|+\left\|V_{1}^{(n)}\right\|\right\}<d-c
$$

If, in addition, $V_{0} \leq V_{1}$, then

$$
\lambda_{k}\left(\left.\left(A+V_{0}\right)\right|_{\operatorname{Ran} E_{A+V_{0}}\left(\left[d-\left\|V_{0}^{(n)}\right\|, \infty\right)\right)}\right) \leq \lambda_{k}\left(\left.\left(A+V_{1}\right)\right|_{\operatorname{Ran} E_{A+V_{1}}\left(\left[d-\left\|V_{1}\right\|^{(n)}, \infty\right)\right)}\right)
$$

for $k \leq \operatorname{dim} \operatorname{Ran} E_{A}([d, \infty))=\operatorname{dim} \operatorname{Ran} E_{A+V_{j}}\left(\left[d-\left\|V_{j}^{(n)}\right\|, \infty\right)\right), j \in\{0,1\}$.

Corollary 2.4 Let $A$ and $V$ be as in Proposition 2.1. Then, the open interval $\left(c+\left\|V^{(p)}\right\|, d-\left\|V^{(n)}\right\|\right)$ belongs to the resolvent set of every $A+t V, t \in[0,1]$, and for each $k \leq \operatorname{dim} \operatorname{Ran} E_{A}([d, \infty))=\operatorname{dim} \operatorname{Ran} E_{A+t V}\left(\left[d-t\left\|V^{(n)}\right\|, \infty\right)\right), t \in[0,1]$, the mapping

$$
[0,1] \ni t \mapsto \lambda_{k}\left(\left.(A+t V)\right|_{\operatorname{Ran} E_{A+t V}\left(\left[d-t\left\|V^{(n)}\right\|, \infty\right)\right)}\right)
$$

is Lipschitz continuous with Lipschitz constant $\|V\|$.

Proof Taking into account that

$$
\langle x,(A+s V) x\rangle-|t-s|\|V\| \leq\langle x,(A+t V) x\rangle \leq\langle x,(A+s V) x\rangle+|t-s|\|V\|
$$

for all $x \in \operatorname{Dom}(A)$, the claim follows immediately from Proposition 2.1. 
It should again be mentioned that the above statements include the particular cases where the norm of the perturbations is less than $(d-c) / 2$ or where the perturbations are semidefinite with a norm less than $d-c$. These cases have essentially been discussed in [27]. There, especially lower bounds on the movement of eigenvalues in gaps of the essential spectrum under certain conditions and the behaviour of edges of the essential spectrum have been studied. However, since this is not the main focus of the present work, this is not pursued further here.

As a consequence of Theorem 1.4, we obtain the following lower bound for the minimax values in the setting of off-diagonal operator perturbations.

Corollary 2.5 In the situation of Theorem 1.4, we have

$$
\lambda_{k}\left(\left.A\right|_{\operatorname{Ran} P_{+}}\right) \leq \lambda_{k}\left(\left.B\right|_{\operatorname{Ran} Q_{+}}\right)
$$

for all $k \leq \operatorname{dim} \operatorname{Ran} P_{+}=\operatorname{dim} \operatorname{Ran} Q_{+}$.

Proof Let $\mathfrak{M}_{+} \subset \mathcal{D}_{+}$with $\operatorname{dim} \mathfrak{M}_{+}=k$. Since $\langle x, V x\rangle=0$ for all $x \in \mathcal{D}_{+}$by hypothesis, we have

$$
\sup _{\substack{x \in \mathfrak{M}_{+} \\\|x\|=1}}\langle x, A x\rangle=\sup _{\substack{x \in \mathfrak{M}_{+} \\\|x\|=1}}\langle x,(A+V) x\rangle \leq \sup _{\substack{x \in \mathfrak{M}_{+} \oplus \mathcal{D}_{-} \\\|x\|=1}}\langle x,(A+V) x\rangle .
$$

Taking the infimum over all such subspaces $\mathfrak{M}_{+}$proves the claim by Theorem 1.4 and the standard minimax values for $\left.A\right|_{\operatorname{Ran} P_{+}}$.

As in Corollary 2.4, we also obtain a continuity statement in the situation of Theorem 1.4 with bounded off-diagonal perturbations. Here, however, we do not have to impose any condition on the norm of the perturbation.

Corollary 2.6 Let $A$ and $V$ be as in Theorem 1.4, and suppose that $V$ is bounded. Then, for each $k \leq \operatorname{dim} \operatorname{Ran} E_{A}((0, \infty))=\operatorname{dim} \operatorname{Ran} E_{A+t V}((0, \infty)), t \in \mathbb{R}$, the mapping

$$
\mathbb{R} \ni t \mapsto \lambda_{k}\left(\left.(A+t V)\right|_{\operatorname{Ran} E_{A+t V}((0, \infty))}\right)
$$

is Lipschitz continuous with Lipschitz constant $\|V\|$.

In the particular case where $B$ is semibounded, Theorem 1.5 allows us to extend Corollaries 2.5 and 2.6 to some degree to off-diagonal form perturbations. Recall here, that semiboundedness of $B$ implies that also $A$ is semibounded, see the proof of Theorem 1.5 below.

Corollary 2.7 Assume the hypotheses of Theorem 1.5.

(a) For each $k \in \mathbb{N}$ with $k \leq \operatorname{dim} \operatorname{Ran} P_{+}=\operatorname{dim} \operatorname{Ran} Q_{+}$one has $\lambda_{k}\left(\left.A\right|_{\operatorname{Ran} P_{+}}\right) \leq$ $\lambda_{k}\left(\left.B\right|_{\operatorname{Ran} Q_{+}}\right)$.

(b) Denote for $t \in(-1 / b, 1 / b)$ by $B_{t}$ the self-adjoint operator associated with the form $\mathfrak{b}_{t}:=\mathfrak{a}+t \mathfrak{v}$ with form domain $\operatorname{Dom}\left[\mathfrak{b}_{t}\right]:=\operatorname{Dom}[\mathfrak{a}]$. Then, for each $k \leq \operatorname{dim} \operatorname{Ran} E_{A}((0, \infty))=\operatorname{dim} \operatorname{Ran} E_{B_{t}}((0, \infty))$, the mapping

$$
(-1 / b, 1 / b) \ni t \mapsto \lambda_{k}\left(\left.B_{t}\right|_{\operatorname{Ran} E_{B_{t}}((0, \infty))}\right)
$$


is locally Lipschitz continuous.

Proof(a). Taking into account that $\mathfrak{v}[x, x]=0$ for all $x \in \mathfrak{D}_{+}$by hypothesis, the inequality $\lambda_{k}\left(\left.A\right|_{\operatorname{Ran} P_{+}}\right) \leq \lambda_{k}\left(\left.B\right|_{\operatorname{Ran} Q_{+}}\right)$is proved by means of Theorem 1.5 in a way analogous to Corollary 2.5 .

(b). Recall that each $B_{t}$ is indeed a semibounded self-adjoint operator with Dom $\left[\mathfrak{b}_{t}\right]=$ $\operatorname{Dom}\left(\left|B_{t}\right|^{1 / 2}\right)$ by the well-known KLMN theorem, and note that each $t \mathfrak{v}$ satisfies the hypotheses of Theorem 1.5 .

Pick $t, s \in(-1 / b, 1 / b)$ with $b|t-s| \leq 1-b|s|$.

Consider first the case where $A$ (and hence $\mathfrak{a}$ ) is lower semibounded with lower bound $m \in \mathbb{R}$. We then have $|\mathfrak{a}[x, x]| \leq \mathfrak{a}[x, x]+(|m|-m)\|x\|^{2}$ for all $x \in$ $\operatorname{Dom}[\mathfrak{a}]$. With $\tilde{a}:=a+b|m|-b m$, this gives

$$
|\mathfrak{v}[x, x]| \leq \tilde{a}\|x\|^{2}+b \mathfrak{a}[x, x] \leq \tilde{a}\|x\|^{2}+b \mathfrak{b}_{s}[x, x]+b|s||\mathfrak{v}[x, x]|
$$

and, hence,

$$
|\mathfrak{v}[x, x]| \leq \frac{\tilde{a}}{1-b|s|}\|x\|^{2}+\frac{b}{1-b|s|} \mathfrak{b}_{s}[x, x]
$$

for all $x \in \operatorname{Dom}[\mathfrak{a}]=\operatorname{Dom}\left[\mathfrak{b}_{s}\right]$. Since $\mathfrak{b}_{t}=\mathfrak{b}_{s}+(t-s) \mathfrak{v}$, we thus obtain

$$
-\frac{\tilde{a}|t-s|}{1-b|s|}+\left(1-\frac{b|t-s|}{1-b|s|}\right) \mathfrak{b}_{s} \leq \mathfrak{b}_{t} \leq \frac{\tilde{a}|t-s|}{1-b|s|}+\left(1+\frac{b|t-s|}{1-b|s|}\right) \mathfrak{b}_{s} .
$$



$-\frac{\tilde{a}|t-s|}{1-b|s|}+\left(1-\frac{b|t-s|}{1-b|s|}\right) \lambda_{k}(s) \leq \lambda_{k}(t) \leq \frac{\tilde{a}|t-s|}{1-b|s|}+\left(1+\frac{b|t-s|}{1-b|s|}\right) \lambda_{k}(s)$

and, therefore,

$$
\left|\lambda_{k}(t)-\lambda_{k}(s)\right| \leq \frac{\tilde{a}|t-s|}{1-b|s|}+\frac{b|t-s|}{1-b|s|}\left|\lambda_{k}(s)\right|
$$

This proves that $t \mapsto \lambda_{k}(t)$ is continuous on $(-1 / b, 1 / b)$ and, in particular, bounded on every compact subinterval of $(-1 / b, 1 / b)$. In turn, it then easily follows from (2.4) that this mapping is even locally Lipschitz continuous, which concludes the case where $A$ is lower semibounded.

If $A$ is upper semibounded with upper bound $m \in \mathbb{R}$, we proceed similarly. We then have $|\mathfrak{a}[x, x]| \leq-\mathfrak{a}[x, x]+(m+|m|)\|x\|^{2}$ for all $x \in \operatorname{Dom}[\mathfrak{a}]$. With $\tilde{a}:=a+b m+b|m|$, this leads to

$$
|\mathfrak{v}[x, x]| \leq \frac{\tilde{a}}{1-b|s|}\|x\|^{2}-\frac{b}{1-b|s|} \mathfrak{b}_{s}[x, x]
$$


for all $x \in \operatorname{Dom}[\mathfrak{a}]=\operatorname{Dom}\left[\mathfrak{b}_{s}\right]$. Analogously as above, we then eventually obtain again (2.4), which proves the claim in the case where $A$ is upper semibounded. This completes the proof.

Remark 2.8 In part (a) of Corollary 2.7, one can also give an upper bound for $\lambda_{k}\left(\left.B\right|_{\operatorname{Ran} Q_{+}}\right)$in terms of the form bounds of $\mathfrak{v}$ : If $A$ is lower semibounded with lower bound $m \in \mathbb{R}$, then we have as in the proof of part (b) of Corollary 2.7 that

$$
|\mathfrak{v}[x, x]| \leq(a+b|m|-b m)\|x\|^{2}+b \mathfrak{a}[x, x]
$$

for all $x \in \operatorname{Dom}[\mathfrak{a}]$, leading to

$$
\lambda_{k}\left(\left.B\right|_{\operatorname{Ran} Q_{+}}\right) \leq(1+b) \lambda_{k}\left(\left.A\right|_{\operatorname{Ran} P_{+}}\right)+(a+b|m|-b m)
$$

for all $k \leq \operatorname{dim} \operatorname{Ran} P_{+}=\operatorname{dim} \operatorname{Ran} Q_{+}$. Similarly, if $A$ is upper semibounded with upper bound $m \in \mathbb{R}$, we have

$$
|\mathfrak{v}[x, x]| \leq(a+b|m|+b m)\|x\|^{2}-b \mathfrak{a}[x, x]
$$

for all $x \in \operatorname{Dom}[\mathfrak{a}]$. If, in addition, $b \leq 1$, this then leads to

$$
\lambda_{k}\left(\left.B\right|_{\operatorname{Ran} Q_{+}}\right) \leq(1-b) \lambda_{k}\left(\left.A\right|_{\operatorname{Ran} P_{+}}\right)+(a+b|m|+b m)
$$

for all $k \leq \operatorname{dim} \operatorname{Ran} P_{+}=\operatorname{dim} \operatorname{Ran} Q_{+}$.

\section{An Example: The Stokes Operator}

We now briefly revisit the Stokes operator in the framework of Theorem 1.5. Here, we mainly rely on [15], but the reader is referred also to [14,Section 7], [29, Chapter 5], [10], and the references cited therein.

Let $\Omega \subset \mathbb{R}^{n}, n \geq 2$, be a bounded domain with $C^{2}$-boundary, and let $v>0$ and $v_{*} \geq 0$. On the Hilbert space $\mathcal{H}=\mathcal{H}_{+} \oplus \mathcal{H}_{-}$with $\mathcal{H}_{+}=L^{2}(\Omega)^{n}$ and $\mathcal{H}_{-}=L^{2}(\Omega)$, we consider the closed, densely defined, and nonnegative form $\mathfrak{a}$ with $\operatorname{Dom}[\mathfrak{a}]:=$ $H_{0}^{1}(\Omega)^{n} \oplus L^{2}(\Omega)$ and

$$
\mathfrak{a}[v \oplus q, u \oplus p]:=v \sum_{j=1}^{n} \int_{\Omega}\left\langle\partial_{j} v(x), \partial_{j} u(x)\right\rangle_{\mathbb{C}^{n}} \mathrm{~d} x
$$

for $u \oplus p, v \oplus q \in \operatorname{Dom}[\mathfrak{a}]$. Clearly, $\mathfrak{a}$ is the form associated to the nonnegative self-adjoint operator $A:=-v \boldsymbol{\Delta} \oplus 0$ on the Hilbert space $\mathcal{H}=\mathcal{H}_{+} \oplus \mathcal{H}_{-}$with $\operatorname{Dom}(A):=\left(H^{2}(\Omega) \cap H_{0}^{1}(\Omega)\right)^{n} \oplus L^{2}(\Omega)$ and $\operatorname{Dom}\left(|A|^{1 / 2}\right)=\operatorname{Dom}[\mathfrak{a}]$, where $\boldsymbol{\Delta}=$ $\Delta \cdot I_{\mathbb{C}^{n}}$ is the vector-valued Dirichlet Laplacian on $\Omega$. Moreover, $P_{+}:=\mathrm{E}_{A}((0, \infty))$ and $P_{-}:=\mathrm{E}_{A}((-\infty, 0])=\mathrm{E}_{A}(\{0\})$ are the orthogonal projections onto $\mathcal{H}_{+}$and $\mathcal{H}_{-}$, respectively. In particular, we have

$$
\mathfrak{D}_{+}:=\operatorname{Ran} P_{+} \cap \operatorname{Dom}\left(|A|^{1 / 2}\right)=H_{0}^{1}(\Omega)^{n} \oplus 0
$$


and

$$
\mathfrak{D}_{-}:=\operatorname{Ran} P_{-} \cap \operatorname{Dom}\left(|A|^{1 / 2}\right)=0 \oplus L^{2}(\Omega) \text {. }
$$

Define the symmetric sesquilinear form $\mathfrak{v}$ on $\mathcal{H}=\mathcal{H}_{+} \oplus \mathcal{H}_{-}$with domain $\operatorname{Dom}[\mathfrak{v}]:=\operatorname{Dom}[\mathfrak{a}]$ by

$$
\mathfrak{v}[v \oplus q, u \oplus p]:=-v_{*}\langle\operatorname{div} v, p\rangle_{L^{2}(\Omega)}-v_{*}\langle q, \operatorname{div} u\rangle_{L^{2}(\Omega)}
$$

for $u \oplus p, v \oplus q \in \operatorname{Dom}[\mathfrak{a}]$. One can show that $v\|\operatorname{div} u\|_{L^{2}(\Omega)}^{2} \leq \mathfrak{a}[u \oplus 0, u \oplus 0]$ for all $u \in \mathfrak{D}_{+}=H_{0}^{1}(\Omega)^{n}$, see, e.g., [29,Proof of Theorem 5.12]. Using Young's inequality, this then implies that $\mathfrak{v}$ is infinitesimally form bounded with respect to $\mathfrak{a}$, see [29,Remark 5.1.3]; cf. also [15,Section 2]. Indeed, for $\varepsilon>0$ and $f=u \oplus p \in \operatorname{Dom}[\mathfrak{a}]$ we obtain

$$
\begin{aligned}
|\mathfrak{v}[f, f]| & \leq 2 v_{*}\left|\langle p, \operatorname{div} u\rangle_{L^{2}(\Omega)}\right| \leq 2 v_{*}\|p\|_{L^{2}(\Omega)}\|\operatorname{div} u\|_{L^{2}(\Omega)} \\
& \leq \varepsilon v\|\operatorname{div} u\|_{L^{2}(\Omega)}^{2}+\varepsilon^{-1} v^{-1} v_{*}^{2}\|p\|_{L^{2}(\Omega)}^{2} \\
& \leq \varepsilon \mathfrak{a}[u \oplus 0, u \oplus 0]+\varepsilon^{-1} v^{-1} v_{*}^{2}\|f\|_{\mathcal{H}}^{2} \\
& =\varepsilon \mathfrak{a}[f, f]+\varepsilon^{-1} v^{-1} v_{*}^{2}\|f\|_{\mathcal{H}}^{2} .
\end{aligned}
$$

Thus, by the well-known KLMN theorem, the form $\mathfrak{b}_{S}:=\mathfrak{a}+\mathfrak{v}$ with $\operatorname{Dom}\left[\mathfrak{b}_{S}\right]=$ $\operatorname{Dom}[\mathfrak{a}]=\operatorname{Dom}\left(|A|^{1 / 2}\right)$ is associated to a unique lower semibounded self-adjoint operator $B_{S}$ on $\mathcal{H}$ with $\operatorname{Dom}\left(\left|B_{S}\right|^{1 / 2}\right)=\operatorname{Dom}\left(|A|^{1 / 2}\right)$, the so-called Stokes operator. It is a self-adjoint extension of the (non-closed) upper dominant block operator matrix

$$
\left(\begin{array}{cc}
-v \boldsymbol{\Delta} & v_{*} \operatorname{grad} \\
-v_{*} \operatorname{div} & 0
\end{array}\right)
$$

defined on $\left(H^{2}(\Omega) \cap H_{0}^{1}(\Omega)\right)^{n} \oplus H^{1}(\Omega)$. In fact, the closure of the latter is a selfadjoint operator, see [10,Theorems 3.7 and 3.9], which yields another characterization of the Stokes operator $B_{S}$.

By rescaling, one obtains from [10,Theorem 3.15] that the essential spectrum of $B_{S}$ is given by

$$
\operatorname{spec}_{\mathrm{ess}}\left(B_{S}\right)=\left\{-\frac{v_{*}^{2}}{v},-\frac{v_{*}^{2}}{2 v}\right\},
$$

cf. [15, Remark 2.2]. In particular, the essential spectrum of $B_{S}$ is purely negative. In turn, the positive spectrum of $B_{S}$, that is, $\operatorname{spec}\left(B_{S}\right) \cap(0, \infty)$, is discrete [15, Theorem 2.1 (i)].

The above shows that the hypotheses of Theorem 1.5 are satisfied in this situation, so that we obtain from Theorem 1.5 and Corollary 2.7 the following result.

Proposition 2.9 Let $B_{S}$ be the Stokes operator as above. Then, the positive spectrum of $B_{S}, \operatorname{spec}\left(B_{S}\right) \cap(0, \infty)$, is discrete, and the positive eigenvalues $\lambda_{k}\left(\left.B_{S}\right|_{\operatorname{Ran} E_{B_{S}}((0, \infty))}\right)$, 
$k \in \mathbb{N}$, of $B_{S}$, enumerated in nondecreasing order and counting multiplicities, admit the representation

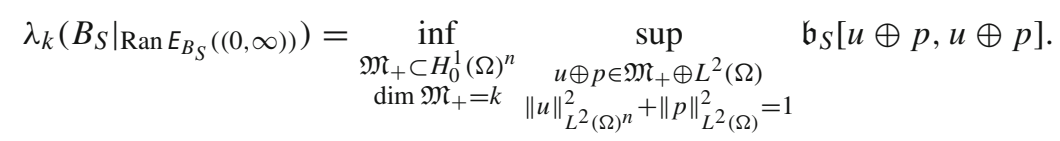

The latter depend locally Lipschitz continuously on $v$ and $v_{*}$ and satisfy the two-sided estimate

$$
v \lambda_{k}(-\boldsymbol{\Delta}) \leq \lambda_{k}\left(B_{S} \mid \operatorname{Ran} E_{B_{S}}((0, \infty))\right) \leq v \lambda_{k}(-\boldsymbol{\Delta})+\frac{v_{*}^{2}}{v}
$$

Proof In view of the above considerations, the representation of the eigenvalues follows from Theorem 1.5, and the lower bound on the eigenvalues follows from Corollary 2.7 (a). Moreover, by rescaling, the continuity statement is a consequence of Corollary 2.7 (b). It remains to show the upper bound on the eigenvalues. To this end, let $\mathfrak{M}_{+} \subset H_{0}^{1}(\Omega)^{n}$ with $\operatorname{dim} \mathfrak{M}_{+}=k \in \mathbb{N}$, and let $f=u \oplus p \in \mathfrak{M}_{+} \oplus L^{2}(\Omega)$ be a normalized vector with $u \neq 0$. Then, $\mu:=\mathfrak{a}[u \oplus 0, u \oplus 0] /\|u\|_{L^{2}(\Omega)^{n}}^{2}=$ $\mathfrak{a}[f, f] /\|u\|_{L^{2}(\Omega)^{n}}^{2}$ is positive and satisfies

$$
\mu \leq \sup _{\substack{v \in \mathfrak{M}_{+} \\\|v\|_{L^{2}(\Omega)^{n}}^{2}=1}} \mathfrak{a}[v \oplus 0, v \oplus 0]
$$

and

$$
\frac{v\|\operatorname{div} u\|_{L^{2}(\Omega)}^{2}}{\mu}=\frac{\|u\|_{L^{2}(\Omega)^{n}}^{2} v\|\operatorname{div} u\|_{L^{2}(\Omega)}^{2}}{\mathfrak{a}[u \oplus 0, u \oplus 0]} \leq\|u\|_{L^{2}(\Omega)^{n}}^{2} \leq 1
$$

Similarly as in (2.5), we now obtain by means of Young's inequality that

$$
\begin{aligned}
|\mathfrak{v}[f, f]| & \leq 2 v_{*}\|p\|_{L^{2}(\Omega)}\|\operatorname{div} u\|_{L^{2}(\Omega)} \leq \mu\|p\|_{L^{2}(\Omega)}^{2}+\frac{v_{*}^{2}\|\operatorname{div} u\|_{L^{2}(\Omega)}^{2}}{\mu} \\
& \leq \mu\|p\|_{L^{2}(\Omega)}^{2}+\frac{v_{*}^{2}}{v}
\end{aligned}
$$

Since $\mathfrak{a}[f, f]=\mu\|u\|_{L^{2}(\Omega)^{n}}^{2}$, this gives

$$
\mathfrak{b}_{S}[f, f] \leq \mathfrak{a}[f, f]+\mu\|p\|_{L^{2}(\Omega)}^{2}+\frac{v_{*}^{2}}{v}=\mu+\frac{v_{*}^{2}}{v}
$$


In light of $\mathfrak{b}_{S}[0 \oplus p, 0 \oplus p]=\mathfrak{a}[0 \oplus p, 0 \oplus p]=0$, we conclude from (2.6) and (2.7) that

$$
\sup _{\substack{u \oplus p \in \mathfrak{M}_{+} \oplus L^{2}(\Omega) \\\|u\|_{L^{2}(\Omega)^{2}}^{2}+\|p\|_{L^{2}(\Omega)}^{2}=1}} \mathfrak{b}_{S}[u \oplus p, u \oplus p] \leq \sup _{\substack{v \in \mathfrak{M}_{+} \\\|v\|_{L^{2}(\Omega)^{n}}^{2}=1}} \mathfrak{a}[v \oplus 0, v \oplus 0]+\frac{v_{*}^{2}}{v},
$$

and taking the infimum over subspaces $\mathfrak{M}_{+} \subset H_{0}^{1}(\Omega)^{n}$ with $\operatorname{dim} \mathfrak{M}_{+}=k$ proves the upper bound. This completes the proof.

Remark 2.10 (1) Choosing $\varepsilon=1$ in (2.5), the upper bound from Remark 2.8 (1) reads

$$
\lambda_{k}\left(\left.B_{S}\right|_{\operatorname{Ran} \mathrm{E}_{B_{S}}((0, \infty))}\right) \leq 2 v \lambda_{k}(-\boldsymbol{\Delta})+\frac{v_{*}^{2}}{v}
$$

for all $k \in \mathbb{N}$, while the choice $\varepsilon=v_{*}$ in (2.5) leads to

$$
\lambda_{k}\left(\left.B_{S}\right|_{\operatorname{Ran} \mathrm{E}_{B_{S}}((0, \infty))}\right) \leq\left(1+v_{*}\right) \nu \lambda_{k}(-\boldsymbol{\Delta})+\frac{v_{*}}{v}
$$

for all $k \in \mathbb{N}$.

(2) For the particular case of $k=1$, a similar upper bound has been established in the proof of [15, Theorem 2.1(i)]:

$$
v \lambda_{1}(-\Delta) \leq \lambda_{1}\left(B_{S} \mid \operatorname{Ran}_{B_{S}}((0, \infty))\right) \leq v \lambda_{1}(-\Delta)+v_{*}\left\|\operatorname{div} u_{0}\right\|_{L^{2}(\Omega)},
$$

where $u_{0} \in\left(H^{2}(\Omega) \cap H_{0}^{1}(\Omega)\right)^{n}$ is a normalized eigenfunction for $-\boldsymbol{\Delta}$ corresponding to the first positive eigenvalue $\lambda_{1}(-\Delta)=\lambda_{1}(-\Delta)$.

Remark 2.11 Since $B_{S}$ is lower semibounded, Proposition 2.9 can alternatively be proved via [12], see Remark 1.6(1). Moreover, since $A=-v \Delta \oplus 0$ has a spectral gap to the right of 0, the same is true with [25], see Remark 1.6(2). In fact, [28] gives for



$$
\lambda_{k}=\inf _{\substack{\mathfrak{M}_{+} \subset\left(H^{2}(\Omega) \cap H_{0}^{1}(\Omega)\right)^{n} \\ \operatorname{dim} \mathfrak{M}_{+}=k}} \sup _{\substack{u \oplus p \in \mathfrak{M}_{+} \oplus H^{1}(\Omega) \\\|u\|_{L^{2}(\Omega)^{n}}^{2}+\|p\|_{L^{2}(\Omega)}^{2}=1}}\left\langle u \oplus p, B_{S}(u \oplus p)\right\rangle .
$$

\section{Reducing to the Off-diagonal Framework}

Apart from the Stokes operator from the previous subsection, the consideration of off-diagonal perturbations in Theorems 1.4 and 1.5 may seem a bit restrictive. However, such perturbations naturally appear when the perturbation is decomposed into its diagonal and off-diagonal part. If the diagonal perturbation can then be handled efficiently in a suitable way, the discussed off-diagonal framework can be applied to the remaining part of the perturbation. The following result makes this precise in the setting of operator perturbations. 
Proposition 2.12 Assume Hypothesis 1.1. Suppose, in addition, that B is of the form $B=A+V, \operatorname{Dom}(B)=\operatorname{Dom}(A)$, with some symmetric A-bounded operator $V$. Denote $A_{ \pm}:=\left.A\right|_{\operatorname{Ran} P_{ \pm}}$, and decompose $\left.V\right|_{\operatorname{Dom}(A)}$ as $\left.V\right|_{\operatorname{Dom}(A)}=V_{\mathrm{diag}}+V_{\mathrm{off}}$, where $V_{\text {diag }}=V_{+} \oplus V_{-}$is the diagonal part of $\left.V\right|_{\operatorname{Dom}(A)}$ and $V_{\mathrm{off}}$ is the off-diagonal part of $\left.V\right|_{\operatorname{Dom}(A)}$ with respect to the decomposition $\operatorname{Ran} P_{+} \oplus \operatorname{Ran} P_{-}$. Suppose that the following hold:

(i) $A+V_{\text {diag }}$ is self-adjoint on $\operatorname{Dom}\left(A+V_{\text {diag }}\right)=\operatorname{Dom}(A)$,

(ii) $V_{\text {off }}$ is $\left(A+V_{\text {diag }}\right)$-bounded with $\left(A+V_{\text {diag }}\right)$-bound smaller than 1 ,

(iii) $\sup \operatorname{spec}\left(A_{-}+V_{-}\right) \leq \inf \operatorname{spec}\left(A_{+}+V_{+}\right)$, and

(iv) $\operatorname{Ker}\left(A_{+}+V_{+}-\mu\right)=\{0\}$ with $\mu:=\sup \operatorname{spec}\left(A_{-}+V_{-}\right)$.

Then, setting $Q:=E_{B}((\mu, \infty))$, one has $\operatorname{dim} \operatorname{Ran} P_{+}=\operatorname{dim} \operatorname{Ran} Q$ and

$$
\lambda_{k}(B \mid \operatorname{Ran} Q)=\inf _{\substack{\mathfrak{M}_{+} \subset \mathcal{D}_{+} \\ \operatorname{dim} \mathfrak{M}_{+}=k}} \sup _{\substack{x \in \mathfrak{M}_{+} \oplus \mathcal{D}_{-} \\\|x\|=1}}\langle x, B x\rangle=\inf _{\substack{\mathfrak{M}_{+} \subset \mathfrak{D}_{+} \\ \operatorname{dim} \mathfrak{M}_{+}=k}} \sup _{\substack{x \in \mathfrak{M}_{+} \oplus \mathfrak{D}_{-} \\\|x\|=1}} \mathfrak{b}[x, x]
$$

for all $k \in \mathbb{N}$ with $k \leq \operatorname{dim} \operatorname{Ran} Q$.

Proof By (iii) and (iv) we obviously have

$$
P_{+}=\mathrm{E}_{A+V_{\text {diag }}}((\mu, \infty)) \text { and } P_{-}=\mathrm{E}_{A+V_{\text {diag }}}((-\infty, \mu]) \text {. }
$$

Upon a spectral shift by $\mu$, the claim now follows from Theorem 1.4 with $A, V$, and $Q_{+}$replaced by $A+V_{\text {diag }}, V_{\text {off }}$, and $Q$, respectively.

Remark 2.13 Conditions (iii) and (iv) in Proposition 2.12 are satisfied if $\mu<v:=$ inf $\operatorname{spec}\left(A_{+}+V_{+}\right)$holds. In this case, the interval $(\mu, v)$ belongs to the resolvent set of the operator $A+V_{\text {diag }}$, and by [26, Theorem 1] (cf. also [1, Theorem 2.1]) to the one of $A+V=\left(A+V_{\text {diag }}\right)+V_{\text {off }}$ as well. In particular, we have $\mathrm{E}_{A+V}((\mu, \infty))=$ $\mathrm{E}_{A+V}([v, \infty))$. This is the situation encountered in the alternative proof of Proposition 2.1 (b) and the proof of Corollary 2.15 below. However, the conclusions can then alternatively be obtained also via [6, 25], cf. Remark 1.6(2).

Since conditions (i) and (ii) in Proposition 2.12 are clearly satisfied if $V$ is bounded, the above provides an alternative way to prove part (b) of Proposition 2.1:

Alternative proof of Proposition 2.1(b) By spectral shift we may assume without loss of generality that $c<0<d$. We then have $P=P_{+}$with $P_{+}$as in Hypothesis 1.1. Let $A_{ \pm}$and $V_{\text {diag }}$ be defined as in Proposition 2.12, and for $\bullet \in\{p, n\}$ denote by $V_{\text {diag }}^{(\bullet)}=$ $V_{+}^{(\bullet)} \oplus V_{-}^{(\bullet)}$ the diagonal part of $V^{(\bullet)}$. Clearly, we have $V_{ \pm}^{(\bullet)} \geq 0$ and $\left\|V_{ \pm}^{(\bullet)}\right\| \leq\left\|V^{(\bullet)}\right\|$, and $V_{\text {diag }}$ decomposes as $V_{\text {diag }}=V_{\text {diag }}^{(p)}-V_{\text {diag }}^{(n)}=\left(V_{+}^{(p)}-V_{+}^{(n)}\right) \oplus\left(V_{-}^{(p)}-V_{-}^{(n)}\right)$. Now,

$$
A_{-}+V_{-}^{(p)}-V_{-}^{(n)} \leq c+\left\|V^{(p)}\right\| \quad \text { and } \quad d-\left\|V^{(n)}\right\| \leq A_{+}+V_{+}^{(p)}-V_{+}^{(n)}
$$

in the sense of quadratic forms. In light of $c+\left\|V^{(p)}\right\|<d-\left\|V^{(n)}\right\|$ and Remark 2.13, applying Proposition 2.12 proves the claim. 
It is worth to note that an analogous reasoning for part (c) of Proposition 2.1 suffers from similar obstacles as the alternative proof based on $[6,25]$ mentioned in Remark 2.2(2).

If $V$ is not bounded, conditions (i) and (ii) in Proposition 2.12 can still be guaranteed via the well-known Kato-Rellich theorem by means of a sufficiently small $A$-bound of $V$, as the following lemma shows.

Lemma 2.14 In the situation of Proposition 2.12, let $V$ have A-bound smaller than $1 / 2$. Then $V_{\text {diag }}$ and $V_{\text {off }}$ both have A-bound smaller than $1 / 2$, and $V_{\text {off }}$ has $\left(A+V_{\text {diag }}\right)$ bound smaller than 1.

Proof By hypothesis, there are constants $a, b \geq 0, b<1 / 2$ such that we have $\|V x\| \leq$ $a\|x\|+b\|A x\|$ for all $x \in \operatorname{Dom}(A)$. Using Young's inequality, this gives for every $\varepsilon>0$ that

$$
\|V x\|^{2} \leq(a\|x\|+b\|A x\|)^{2} \leq a^{2}\left(1+\frac{1}{\varepsilon}\right)\|x\|^{2}+b^{2}(1+\varepsilon)\|A x\|^{2}
$$

for all $x \in \operatorname{Dom}(A)$; cf. [17, Section V.4.1]. Since $A P_{ \pm} x=P_{ \pm} A x$ for all $x \in \operatorname{Dom}(A)$ and the ranges of $P_{ \pm}$are orthogonal, this implies that

$$
\begin{aligned}
\left\|V_{\text {diag }} x\right\|^{2} & =\left\|P_{+} V P_{+} x\right\|^{2}+\left\|P_{-} V P_{-} x\right\|^{2} \\
& \leq a^{2}\left(1+\frac{1}{\varepsilon}\right)\|x\|^{2}+b^{2}(1+\varepsilon)\|A x\|^{2}
\end{aligned}
$$

for all $x \in \operatorname{Dom}(A)$. We choose $\varepsilon>0$ such that $\beta:=b(1+\varepsilon)^{1 / 2}<1 / 2$ and set $\alpha:=a(1+1 / \varepsilon)^{1 / 2}$. It then follows from the above that

$$
\left\|V_{\text {diag }} x\right\| \leq \alpha\|x\|+\beta\|A x\| \quad \text { for all } x \in \operatorname{Dom}(A)
$$

and analogously the same for $V_{\text {off }}$. This shows that $V_{\text {diag }}$ and $V_{\text {off }}$ indeed have $A$-bound smaller than $1 / 2$.

Using standard arguments as, for instance, in [35, Lemma 2.1.6], we obtain

$$
\left\|V_{\text {diag }} x\right\| \leq \frac{\alpha}{1-\beta}\|x\|+\frac{\beta}{1-\beta}\left\|\left(A+V_{\text {diag }}\right) x\right\| \quad \text { for all } x \in \operatorname{Dom}(A)
$$

and, in turn,

$$
\begin{aligned}
\left\|V_{\text {off }} x\right\| & \leq \alpha\|x\|+\beta\|A x\| \leq \alpha\|x\|+\beta\left\|\left(A+V_{\text {diag }}\right) x\right\|+\beta\left\|V_{\text {diag }} x\right\| \\
& \leq\left(\alpha+\frac{\alpha \beta}{1-\beta}\right)\|x\|+\left(\beta+\frac{\beta^{2}}{1-\beta}\right)\left\|\left(A+V_{\text {diag }}\right) x\right\| \\
& =\frac{\alpha}{1-\beta}\|x\|+\frac{\beta}{1-\beta}\left\|\left(A+V_{\text {diag }}\right) x\right\|
\end{aligned}
$$

for all $x \in \operatorname{Dom}\left(A+V_{\text {diag }}\right)=\operatorname{Dom}(A)$, where $\beta /(1-\beta)<1$. This shows that $V_{\text {off }}$ has $\left(A+V_{\text {diag }}\right)$-bound smaller than 1 and, hence, completes the proof. 
A suitable smallness assumption on the perturbation may also be used to guarantee condition (iii) and (iv) in Proposition 2.12 in the sense of Remark 2.13 if the unperturbed operator has a gap in the spectrum. This is demonstrated in the following corollary to the above.

Corollary 2.15 Let $A,(c, d)$, and $\mathcal{D}_{ \pm}$be as in Proposition 2.1, and let $V$ be a symmetric operator that is A-bounded with A-bound smaller than 1/2. Suppose, in addition, that $c<0<d$, and define $A_{ \pm}$and $V_{ \pm}$as in Proposition 2.12. Suppose that there are constants $a_{ \pm}, b_{ \pm} \geq 0, b_{ \pm}<1$, with

$$
\left|\left\langle x, V_{ \pm} x\right\rangle\right| \leq a_{ \pm}\|x\|^{2} \pm b_{ \pm}\left\langle x, A_{ \pm} x\right\rangle \quad \text { for all } x \in \mathcal{D}_{ \pm}
$$

and

$$
a_{+}+a_{-}+b_{+} d-b_{-} c<d-c \text {. }
$$

Then, the interval $\left(a_{-}+\left(1-b_{-}\right) c,\left(1-b_{+}\right) d-a_{+}\right)$belongs to the resolvent set of $A+V$, and one has $\operatorname{dim} \operatorname{Ran} E_{A}([d, \infty))=\operatorname{dim} E_{A+V}\left(\left[\left(1-b_{+}\right) d-a_{+}, \infty\right)\right)$ and

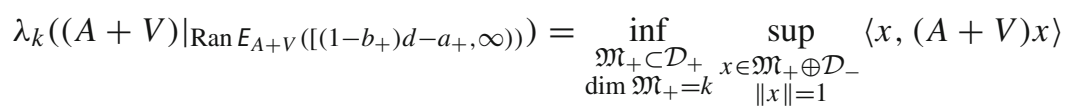

for all $k \in \mathbb{N}$ with $k \leq \operatorname{dim} \operatorname{Ran} E_{A+V}\left(\left[\left(1-b_{+}\right) d-a_{+}, \infty\right)\right)$.

Proof By Lemma 2.14 and the Kato-Rellich theorem, conditions (i) and (ii) in Proposition 2.12 are satisfied. By (2.8) we have

$$
A_{-}+V_{-} \leq a_{-}+\left(1-b_{-}\right) c \quad \text { and } \quad\left(1-b_{+}\right) d-a_{+} \leq A_{+}+V_{+}
$$

in the sense of quadratic forms. Since $a_{-}+\left(1-b_{-}\right) c<\left(1-b_{+}\right) d-a_{+}$by (2.9), the claim now again follows from Proposition 2.12 and Remark 2.13.

Remark 2.16 (1) It is easy to see that the left-hand side of (2.9) is invariant under a spectral shift in $A$. In this respect, an analogous statement as in Corollary 2.15 holds for arbitrary spectral gaps $(c, d)$, not just ones satisfying $c<0<d$.

(2) It follows from Lemma 2.14 that $V_{ \pm}$is $A_{ \pm}$-bounded with $A_{ \pm}$-bound smaller than $1 / 2$. In turn, since both $A_{ \pm}$are semibounded, constants $a_{ \pm}, b_{ \pm} \geq 0, b_{ \pm}<1 / 2$, satisfying (2.8) always exist by [17,Theorem VI.1.38]. Condition (2.9) can then be guaranteed for $t V$ instead of $V$ for $t \in \mathbb{R}$ with sufficiently small modulus.

(3) As indicated in Remark 2.13, Corollary 2.15 can also be proved via $[6,25]$ by verifying the operator analogue to (1.1). In fact, that approach even allows to weaken the assumption on the $A$-bound of $V$ from being smaller than $1 / 2$ to merely being smaller than 1 since it is then not necessary to have that $V_{\text {off }}$ has $\left(A+V_{\text {diag }}\right)$-bound smaller than 1 . 


\section{An Abstract Minimax Principle in Spectral Gaps}

We rely on the following abstract minimax principle in spectral gaps, part (a) of which is extracted from [11] and part (b) of which is its natural adaptation to the operator framework; cf. also [27,Proposition A.3]. For the convenience of the reader, its proof is reproduced in Appendix A below.

Proposition 3.1 Assume Hypothesis 1.1.

(a) If we have $\operatorname{Dom}\left(|B|^{1 / 2}\right)=\operatorname{Dom}\left(|A|^{1 / 2}\right), \mathfrak{b}[x, x] \leq 0$ for all $x \in \mathfrak{D}_{-}$, and $\operatorname{Ran}\left(P_{+} Q_{+} \mid \mathfrak{D}_{+}\right) \supset \mathfrak{D}_{+}$, then

$$
\lambda_{k}\left(\left.B\right|_{\operatorname{Ran} Q_{+}}\right)=\inf _{\substack{\mathfrak{M}_{+} \subset \mathfrak{D}_{+} \\ \operatorname{dim}\left(\mathfrak{M}_{+}\right)=k}} \sup _{\substack{x \in \mathfrak{M}_{+} \oplus \mathfrak{D}_{-} \\\|x\|=1}} \mathfrak{b}[x, x]
$$

for all $k \in \mathbb{N}$ with $k \leq \operatorname{dim} \operatorname{Ran} P_{+}$.

(b) If we have $\operatorname{Dom}(B)=\operatorname{Dom}(A),\langle x, B x\rangle \leq 0$ for all $x \in \mathcal{D}_{-}$, and $\operatorname{Ran}\left(P_{+} Q_{+} \mid \mathcal{D}_{+}\right) \supset \mathcal{D}_{+}$, then

$$
\lambda_{k}\left(\left.B\right|_{\left.\operatorname{Ran} Q_{+}\right)=} \inf _{\substack{\mathfrak{M}_{+} \subset \mathcal{D}_{+} \\ \operatorname{dim}\left(\mathfrak{M}_{+}\right)=k}} \sup _{\substack{x \in \mathfrak{M}_{+} \oplus \mathcal{D}_{-} \\\|x\|=1}}\langle x, B x\rangle\right.
$$

for all $k \in \mathbb{N}$ with $k \leq \operatorname{dim} \operatorname{Ran} P_{+}$.

Remark 3.2 The above proposition is tailored towards spectral gaps to the right of 0 , but by a spectral shift we can of course handle also spectral gaps to the right of any point $\gamma \in \mathbb{R}$. Indeed, we have $\mathrm{E}_{A-\gamma}((0, \infty))=\mathrm{E}_{A}((\gamma, \infty))$ for $\gamma \in \mathbb{R}$ and analogously for $B$. Moreover, the form associated to the operator $B-\gamma$ is known to agree with the form $\mathfrak{b}-\gamma$. The latter can be seen for instance with an analogous reasoning as in [31,Proposition 10.5 (a)]; cf. also Lemma B.6 in Appendix B below.

Remark 3.3 Since $P_{+}$and $Q_{+}$are spectral projections for the respective operators, we have $\operatorname{Ran}\left(P_{+} Q_{+} \mid \mathfrak{D}_{+}\right) \subset \mathfrak{D}_{+}$and $\operatorname{Ran}\left(P_{+} Q_{+} \mid \mathcal{D}_{+}\right) \subset \mathcal{D}_{+}$. In this respect, the condition $\operatorname{Ran}\left(\left.P_{+} Q_{+}\right|_{\mathfrak{D}_{+}}\right) \supset \mathfrak{D}_{+}$in part (a) of Proposition 3.1 actually means that the restriction $P_{+} Q_{+} \mid \mathfrak{D}_{+}: \mathfrak{D}_{+} \rightarrow \mathfrak{D}_{+}$is surjective. This has not been formulated explicitly in the statement of [11,Theorem 1] but has instead been guaranteed by the stronger condition

$$
\left\|(|A|+I)^{1 / 2} P_{+} Q_{-}(|A|+I)^{-1 / 2}\right\|<1 .
$$

In fact, taking into account that $\mathfrak{D}_{+}=\operatorname{Ran}\left(\left.(|A|+I)^{-1 / 2}\right|_{\operatorname{Ran} P_{+}}\right)$, a standard Neumann series argument in the Hilbert space Ran $P_{+}$then even gives bijectivity of the restriction $\left.P_{+} Q_{+}\right|_{\mathfrak{D}_{+}}$, see Step 2 of the proof of [11, Theorem 1]. In this reasoning, the operators $(|A|+I)^{ \pm 1 / 2}$ can be replaced by $(|A|+\alpha I)^{ \pm 1 / 2}$ for any $\alpha>0$; if $|A|$ has a bounded inverse, also $\alpha=0$ can be considered here.

Of course, the above reasoning also applies in the situation of part (b) of Proposition 3.1, but with $(|A|+\alpha I)^{ \pm 1 / 2}$ replaced by $(|A|+\alpha I)^{ \pm 1}$. 
In the context of our main theorems, the restriction $\left.P_{+} Q_{+}\right|_{\operatorname{Ran} P_{+}}$, understood as an endomorphism of Ran $P_{+}$, will always be bijective, cf. Remark 3.5 (1) below. It turns out that then the hypotheses of part (b) in Proposition 3.1 imply those of part (a), in which case both representations for the minimax values in Proposition 3.1 are valid. More precisely, we have the following lemma, essentially based on the well-known Heinz inequality, cf. Appendix B below.

Lemma 3.4 Assume Hypothesis 1.1 with $\operatorname{Dom}(A)=\operatorname{Dom}(B)$.

(a) One has $\operatorname{Dom}\left(|A|^{1 / 2}\right)=\operatorname{Dom}\left(|B|^{1 / 2}\right)$.

(b) If $\langle x, B x\rangle \leq 0$ for all $x \in \mathcal{D}_{-}$, then $\mathfrak{b}[x, x] \leq 0$ for all $x \in \mathfrak{D}_{-}$.

(c) If the restriction $\left.P_{+} Q_{+}\right|_{\operatorname{Ran} P_{+}}: \operatorname{Ran} P_{+} \rightarrow \operatorname{Ran} P_{+}$is bijective and $\operatorname{Ran}\left(P_{+} Q_{+} \mid \mathcal{D}_{+}\right) \supset \mathcal{D}_{+}$, then also $\operatorname{Ran}\left(\left.P_{+} Q_{+}\right|_{\mathfrak{D}_{+}}\right) \supset \mathfrak{D}_{+}$.

Proof(a). This is a consequence of the well-known Heinz inequality, see, e.g., Corollary B.3 below. Alternatively, this follows by classical considerations regarding operator and form boundedness, see Remark B.4 below.

(b). It follows from part (a) that the operator $|B|^{1 / 2}\left(|A|^{1 / 2}+I\right)^{-1}$ is closed and everywhere defined, hence bounded by the closed graph theorem. Thus,

$$
\left\||B|^{1 / 2} x\right\| \leq\left\||B|^{1 / 2}\left(|A|^{1 / 2}+I\right)^{-1}\right\| \cdot\left\|\left(|A|^{1 / 2}+I\right) x\right\|
$$

for all $x \in \operatorname{Dom}\left(|A|^{1 / 2}\right)=\operatorname{Dom}\left(|B|^{1 / 2}\right)$. Since $\mathcal{D}_{-}$is a core for the operator $\left.|A|_{\operatorname{Ran} P_{-}}\right|^{1 / 2}=\left.|A|^{1 / 2}\right|_{\operatorname{Ran} P_{-}}$with $\operatorname{Dom}\left(\left.|A|^{1 / 2}\right|_{\operatorname{Ran} P_{-}}\right)=\mathfrak{D}_{-}$, the inequality $\mathfrak{b}[x, x] \leq 0$ for $x \in \mathfrak{D}_{-}$now follows from the hypothesis $\langle x, B x\rangle \leq 0$ for all $x \in \mathcal{D}_{-}$by approximation.

(c). We clearly have $\operatorname{Ran}\left(P_{+} Q_{+} \mid \mathcal{D}_{+}\right)=\mathcal{D}_{+}, \mathcal{D}_{+}=\operatorname{Dom}\left(\left.A\right|_{\operatorname{Ran} P_{+}}\right)$, and $\mathfrak{D}_{+}=$ $\operatorname{Dom}\left(\left.|A|_{\operatorname{Ran} P_{+}}\right|^{1 / 2}\right)$. Applying Corollary B.5 below with the choices $\Lambda_{1}=\Lambda_{2}=$ $\left.A\right|_{\operatorname{Ran} P_{+}}$and $S=P_{+} Q_{+} \mid \operatorname{Ran} P_{+}$therefore implies that $\operatorname{Ran}\left(P_{+} Q_{+} \mid \mathfrak{D}_{+}\right)=\mathfrak{D}_{+}$, which proves the claim.

Remark 3.5 (1) In light of the identity $P_{+} Q_{+}=P_{+}-P_{+} Q_{-}$, the bijectivity of $\left.P_{+} Q_{+}\right|_{\operatorname{Ran} P_{+}}: \operatorname{Ran} P_{+} \rightarrow \operatorname{Ran} P_{+}$can be guaranteed, for instance, by the condition $\left\|P_{+} Q_{-}\right\|<1$ via a standard Neumann series argument. Since $P_{+}-Q_{+}=$ $P_{+} Q_{-}-P_{-} Q_{+}$and, in particular, $\left\|P_{+} Q_{-}\right\| \leq\left\|P_{+}-Q_{+}\right\|$, this condition holds if the stronger inequality $\left\|P_{+}-Q_{+}\right\|<1$ is satisfied. In the latter case, there also is a unitary operator $U$ with $Q_{+} U=U P_{+}$, see, e.g., [17, Theorem I.6.32], so that automatically $\operatorname{dim} \operatorname{Ran} P_{+}=\operatorname{dim} \operatorname{Ran} Q_{+}$. It is this situation we encounter in Theorems 1.3-1.5.

(2) In the case where $B$ is an infinitesimal operator perturbation of $A$, the inequality $\left\|P_{+} Q_{-}\right\|<1$ already implies that $\operatorname{Ran}\left(P_{+} Q_{+} \mid \mathcal{D}_{+}\right) \supset \mathcal{D}_{+}$, see the following section; the particular case where $B$ is a bounded perturbation of $A$ has previously been considered in [27,Lemma A.6]. For more general, not necessarily infinitesimal, perturbations, this remains so far an open problem. 


\section{Proof of Theorem 1.2: The Graph Norm Approach}

In this section we show that the inequality $\left\|P_{+} Q_{-}\right\|<1$ in the context of Theorem 1.2 implies that $\operatorname{Ran}\left(P_{+} Q_{+} \mid \mathcal{D}_{+}\right) \supset \mathcal{D}_{+}$, which is essentially what is needed to deduce Theorem 1.2 from Proposition 3.1 and Lemma 3.4. The main technique used to accomplish this can in fact be formulated in a much more general framework:

Recall that for a closed operator $\Lambda$ on a Banach space with norm $\|\cdot\|$, its domain $\operatorname{Dom}(\Lambda)$ can be equipped with the graph norm

$$
\|x\|_{\Lambda}:=\|x\|+\|\Lambda x\|, \quad x \in \operatorname{Dom}(\Lambda)
$$

which makes $\left(\operatorname{Dom}(\Lambda),\|\cdot\|_{\Lambda}\right)$ a Banach space. Also recall that a linear operator $K$ with $\operatorname{Dom}(K) \supset \operatorname{Dom}(\Lambda)$ is called $\Lambda$-bounded with $\Lambda$-bound $\beta_{*} \geq 0$ if for all $\beta>\beta_{*}$ there is an $\alpha \geq 0$ with

$$
\|K x\| \leq \alpha\|x\|+\beta\|\Lambda x\| \quad \text { for all } x \in \operatorname{Dom}(\Lambda)
$$

and if there is no such $\alpha$ for $0<\beta<\beta_{*}$.

The following lemma extends part (a) of [27,Proposition A.5], taken from Lemma 3.9 in the author's Ph.D. thesis [32], to relatively bounded commutators.

Lemma 4.1 Let $\Lambda$ be a closed operator on a Banach space, $K$ be $\Lambda$-bounded with $\Lambda$-bound $\beta_{*} \geq 0$, and let $S$ be bounded with $\operatorname{Ran}\left(\left.S\right|_{\operatorname{Dom}(\Lambda)}\right) \subset \operatorname{Dom}(\Lambda)$ and

$$
\Lambda S x-S \Lambda x=K x \text { for all } x \in \operatorname{Dom}(\Lambda)
$$

Then, the restriction $\left.S\right|_{\operatorname{Dom}(\Lambda)}$ is bounded with respect to the graph norm for $\Lambda$, and the corresponding spectral radius $r_{\Lambda}(S)=\lim _{k \rightarrow \infty}\left\|\left(\left.S\right|_{\operatorname{Dom}(\Lambda)}\right)^{k}\right\|_{\Lambda}^{1 / k}$ satisfies

$$
r_{\Lambda}(S) \leq\|S\|+\beta_{*}
$$

Proof Only small modifications to the reasoning from [32,Lemma 3.9], [27,Proposition A.5] are necessary. For the sake of completeness, we reproduce the full argument here:

Let $\beta>\beta_{*}$ and $\alpha \geq 0$ such that (4.1) holds. Then, for $x \in \operatorname{Dom}(\Lambda)$ one has

$$
\|\Lambda S x\| \leq\|S\|\|\Lambda x\|+\|K x\| \leq(\|S\|+\beta)\|\Lambda x\|+\alpha\|x\|,
$$

so that

$$
\|S x\|_{\Lambda}=\|S x\|+\|\Lambda S x\| \leq(\|S\|+\beta)\|x\|_{\Lambda}+\alpha\|x\| .
$$

In particular, $\left.S\right|_{\operatorname{Dom}(\Lambda)}$ is bounded with respect to the graph norm $\|\cdot\| \Lambda$ with $\|S\|_{\Lambda} \leq$ $\|S\|+\beta+\alpha$. 
Now, a straightforward induction yields

$$
\left\|S^{k} x\right\|_{\Lambda} \leq(\|S\|+\beta)^{k}\|x\|_{\Lambda}+k \alpha(\|S\|+\beta)^{k-1}\|x\|, \quad x \in \operatorname{Dom}(\Lambda),
$$

for $k \in \mathbb{N}$. Hence, $\left\|\left(\left.S\right|_{\operatorname{Dom}(\Lambda)}\right)^{k}\right\|_{\Lambda} \leq(\|S\|+\beta)^{k}+k \alpha(\|S\|+\beta)^{k-1}$, so that

$$
\begin{aligned}
r_{\Lambda}(S) & =\lim _{k \rightarrow \infty}\left\|\left(\left.S\right|_{\operatorname{Dom}(\Lambda)}\right)^{k}\right\|_{\Lambda}^{1 / k} \leq \lim _{k \rightarrow \infty}\left((\|S\|+\beta)^{k}+k \alpha(\|S\|+\beta)^{k-1}\right)^{1 / k} \\
& =\|S\|+\beta
\end{aligned}
$$

Since $\beta>\beta_{*}$ was chosen arbitrarily, this proves the claim.

We are now in position to prove Theorem 1.2.

Proof of Theorem 1.2 We mainly follow the line of reasoning in the proof of [27,Lemma A.6]. Only a few additional considerations are necessary in order to accommodate unbounded perturbations $V$ by means of Lemma 4.1. For convenience of the reader, we nevertheless reproduce the whole argument here.

Define $S, T: \operatorname{Ran} P_{+} \rightarrow \operatorname{Ran} P_{+}$by

$$
S:=P_{+} Q_{-}\left|\operatorname{Ran} P_{+}, \quad T:=P_{+} Q_{+}\right|_{\operatorname{Ran} P_{+}}=I_{\operatorname{Ran} P_{+}}-S .
$$

By hypothesis, we have $\|S\| \leq\left\|P_{+} Q_{-}\right\|<1$, so that $T$ is bijective. In light of Proposition 3.1 and Lemma 3.4, it now remains to show the inclusion $\operatorname{Ran}\left(\left.P_{+} Q_{+}\right|_{\mathcal{D}_{+}}\right) \supset \mathcal{D}_{+}$, that is, $\operatorname{Ran}\left(\left.T^{-1}\right|_{\mathcal{D}_{+}}\right) \subset \mathcal{D}_{+}$. To this end, we rewrite $T^{-1}$ as a Neumann series,

$$
T^{-1}=\left(I_{\text {Ran } P_{+}}-S\right)^{-1}=\sum_{k=0}^{\infty} S^{k} .
$$

Clearly, $S$ maps the domain $\mathcal{D}_{+}=\operatorname{Dom}\left(\left.A\right|_{\operatorname{Ran} P_{+}}\right)$into itself, so that the inclusion $\operatorname{Ran}\left(\left.T^{-1}\right|_{\mathcal{D}_{+}}\right) \subset \mathcal{D}_{+}$holds if the above series converges also with respect to the graph norm for the closed operator $\Lambda:=\left.A\right|_{\operatorname{Ran} P_{+}}$. This, in turn, is the case if the corresponding spectral radius $r_{\Lambda}(S)$ of $S$ is smaller than 1 .

For $x \in \mathcal{D}_{+} \subset$ Ran $P_{+}$we compute

$$
\begin{aligned}
\Lambda S x & =A P_{+} Q_{-} x=P_{+}(A+V) Q_{-} x-P_{+} V Q_{-} x \\
& =P_{+} Q_{-}(A+V) x-P_{+} V Q_{-} x \\
& =S \Lambda x+K x
\end{aligned}
$$

with

$$
K:=\left.\left(P_{+} Q_{-} V-P_{+} V Q_{-}\right)\right|_{\operatorname{Ran} P_{+}}
$$

We show that the operator $K$ is $\Lambda$-bounded with $\Lambda$-bound 0 . Indeed, let $b>0$, and choose $a \geq 0$ with $\|V x\| \leq a\|x\|+b\|A x\|$ for all $x \in \operatorname{Dom}(A)$; recall that $V$ is 
infinitesimal with respect to $A$ by hypothesis. Then,

$$
\left\|V Q_{-} x\right\| \leq a\left\|Q_{-} x\right\|+b\left\|A Q_{-} x\right\| \leq a\|x\|+b\|(A+V) x\|+b\left\|V Q_{-} x\right\|,
$$

so that

$$
\begin{aligned}
\left\|V Q_{-} x\right\| & \leq \frac{a}{1-b}\|x\|+\frac{b}{1-b}(\|A x\|+\|V x\|) \\
& \leq \frac{a(1+b)}{1-b}\|x\|+\frac{b(1+b)}{1-b}\|A x\| .
\end{aligned}
$$

Thus,

$$
\begin{aligned}
\|K x\| & \leq\left\|P_{+} Q_{-}\right\|\|V x\|+\left\|V Q_{-} x\right\| \\
& \leq a\left(\left\|P_{+} Q_{-}\right\|+\frac{1+b}{1-b}\right)\|x\|+b\left(\left\|P_{+} Q_{-}\right\|+\frac{1+b}{1-b}\right)\|\Lambda x\|
\end{aligned}
$$

for $x \in \operatorname{Dom}(\Lambda)=\mathcal{D}_{+}$. Since $b>0$ was chosen arbitrarily, this implies that $K$ is $\Lambda$ bounded with $\Lambda$-bound 0 . It therefore follows from Lemma 4 .1 that $r_{\Lambda}(S) \leq\|S\|<1$, which completes the proof.

Remark 4.2 (1) Estimate (4.2) suggests that also relatively bounded perturbations $V$ that are not necessarily infinitesimal with respect to $A$ can be considered here. Indeed, if $b_{*} \in[0,1)$ is the $A$-bound of $V$, then by (4.2) and Lemma 4.1 we have

$$
r_{\Lambda}(S) \leq\left\|P_{+} Q_{-}\right\|+b_{*}\left(\left\|P_{+} Q_{-}\right\|+\frac{1+b_{*}}{1-b_{*}}\right),
$$

and the right-hand side of the latter is smaller than 1 if and only if

$$
\left\|P_{+} Q_{-}\right\|<\frac{1-2 b_{*}-b_{*}^{2}}{1-b_{*}^{2}}
$$

This is a reasonable condition on the norm $\left\|P_{+} Q_{-}\right\|$only for $b_{*}<\sqrt{2}-1$.

(2) A similar result as in (1) can be obtained in terms of the $(A+V)$-bound of $V$ : If for some $\tilde{b} \in[0,1)$ and $\tilde{a} \geq 0$ one has $\|V x\| \leq \tilde{a}\|x\|+\tilde{b}\|(A+V) x\|$ for all $x \in \operatorname{Dom}(A)=\operatorname{Dom}(A+V)$, then standard arguments as in the above proof of Theorem 1.2 (see also [35,Lemma 2.1.6]) show that

$$
\|V x\| \leq \frac{\tilde{a}}{1-\tilde{b}}\|x\|+\frac{\tilde{b}}{1-\tilde{b}}\|A x\|
$$


and, in turn,

$$
\begin{aligned}
\left\|V Q_{-} x\right\| & \leq \tilde{a}\|x\|+\tilde{b}\|(A+V) x\| \leq \tilde{a}\|x\|+\tilde{b}\|A x\|+\tilde{b}\|V x\| \\
& \leq \tilde{a}\left(1+\frac{\tilde{b}}{1-\tilde{b}}\right)\|x\|+\tilde{b}\left(1+\frac{\tilde{b}}{1-\tilde{b}}\right)\|A x\| \\
& =\frac{\tilde{a}}{1-\tilde{b}}\|x\|+\frac{\tilde{b}}{1-\tilde{b}}\|A x\|
\end{aligned}
$$

for all $x \in \operatorname{Dom}(A)$. Plugging these into (4.2) gives

$$
\begin{aligned}
\|K x\| & \leq\left\|P_{+} Q_{-}\right\|\|V x\|+\left\|V Q_{-} x\right\| \\
& \leq\left(1+\left\|P_{+} Q_{-}\right\|\right)\left(\frac{\tilde{a}}{1-\tilde{b}}\|x\|+\frac{\tilde{b}}{1-\tilde{b}}\|\Lambda x\|\right)
\end{aligned}
$$

for all $x \in \operatorname{Dom}(\Lambda)=\mathcal{D}_{+}$, which eventually leads to

$$
r_{\Lambda}(S) \leq\left\|P_{+} Q_{-}\right\|+\frac{\tilde{b}\left(1+\left\|P_{+} Q_{-}\right\|\right)}{1-\tilde{b}}=\frac{\left\|P_{+} Q_{-}\right\|+\tilde{b}}{1-\tilde{b}}
$$

The right-hand side of the latter is smaller than 1 if and only if

$$
\left\|P_{+} Q_{-}\right\|<1-2 \tilde{b}
$$

which is a reasonable condition on $\left\|P_{+} Q_{-}\right\|$only for $\tilde{b}<1 / 2$.

\section{The Block Diagonalization Approach}

In this section, we discuss an approach to verify the hypotheses of Proposition 3.1 and Lemma 3.4 which relies on techniques previously discussed in the context of block diagonalizations of operators and forms, for instance in [24] and [14], respectively; cf. also Remark 5.4 below.

Recall that for the two orthogonal projections $P_{+}$and $Q_{+}$from Hypothesis 1.1 the inequality $\left\|P_{+}-Q_{+}\right\|<1$ holds if and only if $\operatorname{Ran} Q_{+}$can be represented as

$$
\operatorname{Ran} Q_{+}=\left\{f \oplus X f \mid f \in \operatorname{Ran} P_{+}\right\}
$$

with some bounded linear operator $X:$ Ran $P_{+} \rightarrow$ Ran $P_{-}$; in this case, one has

$$
\left\|P_{+}-Q_{+}\right\|=\frac{\|X\|}{\sqrt{1+\|X\|^{2}}}
$$


see, e.g., [18,Corollary 3.4(i)]. The orthogonal projection $Q_{+}$can then be represented as the $2 \times 2$ block operator matrices

$$
\begin{aligned}
Q_{+} & =\left(\begin{array}{cc}
\left(I_{\operatorname{Ran} P_{+}}+X^{*} X\right)^{-1} & \left(I_{\operatorname{Ran} P_{+}}+X^{*} X\right)^{-1} X^{*} \\
X\left(I_{\operatorname{Ran} P_{+}}+X^{*} X\right)^{-1} & X\left(I_{\operatorname{Ran} P_{+}}+X^{*} X\right)^{-1} X^{*}
\end{array}\right) \\
& =\left(\begin{array}{cc}
\left(I_{\operatorname{Ran} P_{+}}+X^{*} X\right)^{-1} & X^{*}\left(I_{\operatorname{Ran} P_{-}}+X X^{*}\right)^{-1} \\
\left(I_{\operatorname{Ran} P_{-}}+X X^{*}\right)^{-1} X & X X^{*}\left(I_{\operatorname{Ran} P_{-}}+X X^{*}\right)^{-1}
\end{array}\right)
\end{aligned}
$$

with respect to Ran $P_{+} \oplus$ Ran $P_{-}$, see, e.g., [18,Remark 3.6]. In particular, we have

$$
\left.P_{+} Q_{+}\right|_{\operatorname{Ran} P_{+}}=\left(I_{\operatorname{Ran} P_{+}}+X^{*} X\right)^{-1},
$$

which is in fact the starting point for the current approach: With regard to the desired relations $\operatorname{Ran}\left(P_{+} Q_{+} \mid \mathcal{D}_{+}\right) \supset \mathcal{D}_{+}$and $\operatorname{Ran}\left(\left.P_{+} Q_{+}\right|_{\mathfrak{D}_{+}}\right) \supset \mathfrak{D}_{+}$, we need to establish that the operator $I_{\operatorname{Ran} P_{+}}+X^{*} X$ maps $\mathcal{D}_{+}$and $\mathfrak{D}_{+}$into $\mathcal{D}_{+}$and $\mathfrak{D}_{+}$, respectively.

Define the skew-symmetric operator $Y$ via the $2 \times 2$ block operator matrix

$$
Y=\left(\begin{array}{cc}
0 & -X^{*} \\
X & 0
\end{array}\right)
$$

with respect to Ran $P_{+} \oplus \operatorname{Ran} P_{-}$. Then, the operators $I \pm Y$ are bijective with

$$
(I-Y)(I+Y)=\left(\begin{array}{cc}
I_{\operatorname{Ran} P_{+}+X^{*} X} & 0 \\
0 & I_{\operatorname{Ran} P_{-}}+X X^{*}
\end{array}\right) .
$$

The following lemma is extracted from various sources. We comment on this afterwards in Remark 5.2 below.

Lemma 5.1 Suppose that the projections $P_{+}$and $Q_{+}$from Hypothesis 1.1 satisfy $\left\|P_{+}-Q_{+}\right\|<1$, and let the operators $X$ and $Y$ be as in (5.1) and (5.5), respectively. Moreover, let $\mathcal{C}$ be an invariant subspace for both $P_{+}$and $Q_{+}$we have such that $\mathcal{C}=\left(\mathcal{C} \cap \operatorname{Ran} P_{+}\right) \oplus\left(\mathcal{C} \cap \operatorname{Ran} P_{-}\right)=: \mathcal{C}_{+} \oplus \mathcal{C}_{-}$.

Then, the following are equivalent:

(i) $I_{\mathrm{Ran} P_{+}}+X^{*} X$ maps $\mathcal{C}_{+}$into itself;

(ii) $I_{\operatorname{Ran} P_{-}}+X X^{*}$ maps $\mathcal{C}_{-}$into itself;

(iii) $Y$ maps $\mathcal{C}$ into itself;

(iv) $(I+Y)$ maps $\mathcal{C}$ into itself;

(v) $(I-Y)$ maps $\mathcal{C}$ into itself.

Proof Clearly, the hypotheses imply that $P_{+} Q_{+}$maps $\mathcal{C}$ into $\mathcal{C}_{+}$and $P_{-} Q_{+}$maps $\mathcal{C}$ into $\mathcal{C}_{-}$.

(i) $\Rightarrow$ (ii). Let $g \in \mathcal{C}_{-}$. Using the first representation in (5.3), we then have identity $\left(I_{\text {Ran } P_{+}}+X^{*} X\right)^{-1} X^{*} g=\left(P_{+} Q_{+} \mid \operatorname{Ran} P_{-}\right) g \in \mathcal{C}_{+}$. Hence, $X^{*} g \in \mathcal{C}_{+}$by (i) and, in turn, $h:=\left(I_{\operatorname{Ran} P_{+}}+X^{*} X\right) X^{*} g \in \mathcal{C}_{+}$. Using again (5.3), this yields

$$
\begin{aligned}
\left(I_{\operatorname{Ran} P_{-}}+X X^{*}\right) g & =g+X X^{*} g=g+X\left(I_{\operatorname{Ran} P_{+}}+X^{*} X\right)^{-1} h \\
& =g+\left(P_{-} Q_{+} \mid \operatorname{Ran} P_{+}\right) h \in \mathcal{C}_{-} .
\end{aligned}
$$


As a byproduct, we have also shown that $X^{*}$ maps $\mathcal{C}_{-}$into $\mathcal{C}_{+}$.

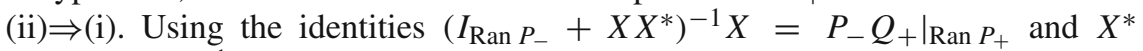
$\left(I_{\text {Ran } P_{-}}+X X^{*}\right)^{-1}=\left.P_{+} Q_{+}\right|_{\operatorname{Ran} P_{-}}$taken from the second representation in (5.3), the proof is completely analogous to the implication (i) $\Rightarrow$ (ii). In particular, we likewise obtain as a byproduct that $X$ maps $\mathcal{C}_{+}$into $\mathcal{C}_{-}$.

(i),(ii) $\Rightarrow$ (iii). We have already seen that $X$ maps $\mathcal{C}_{+}$into $\mathcal{C}_{-}$and that $X^{*}$ maps $\mathcal{C}_{-}$ into $\mathcal{C}_{+}$. Taking into account that $\mathcal{C}=\mathcal{C}_{+} \oplus \mathcal{C}_{-}$, this means that $Y$ maps $\mathcal{C}$ into itself.

(iii) $\Leftrightarrow$ (iv),(v). This is clear.

(iv),(v) $\Rightarrow$ (i),(ii). This follows immediately from identity (5.6).

Remark 5.2 The proof of the equivalence (i) $\Leftrightarrow$ (ii) and the one of the implication (i),(ii) $\Rightarrow$ (iii) in Lemma 5.1 are extracted from the proof of $[14$, Theorem 5.1]; see also [29,Theorem 6.3.1 and Lemma 6.3.3].

The equivalence (iv) $\Leftrightarrow(\mathrm{v})$ can alternatively be directly obtained from the identity

$$
\left(\begin{array}{cc}
I_{\mathrm{Ran}} P_{+} & 0 \\
0 & -I_{\operatorname{Ran} P_{-}}
\end{array}\right)(I+Y)\left(\begin{array}{cc}
I_{\operatorname{Ran} P_{+}} & 0 \\
0 & -I_{\operatorname{Ran} P_{-}}
\end{array}\right)=I-Y .
$$

Such an argument has been used in the proof of [24,Proposition 3.3].

The implication (iv),(v) $\Rightarrow$ (i) can essentially be found in the proof of [14,Theorem 5.1] and [29,Remark 6.3.2].

Below, we apply Lemma 5.1 with $\mathcal{C}=\operatorname{Dom}(A)=\operatorname{Dom}(B)=\mathcal{D}_{+} \oplus \mathcal{D}_{-}$ or $\mathcal{C}=\operatorname{Dom}\left(|A|^{1 / 2}\right)=\operatorname{Dom}\left(|B|^{1 / 2}\right)=\mathfrak{D}_{+} \oplus \mathfrak{D}_{-}$, depending on the situation. The easiest case is encountered in Theorem 1.3, where we are in the semibounded setting:

Proof of Theorem 1.3 Let $\operatorname{Dom}\left(|A|^{1 / 2}\right)=\operatorname{Dom}\left(|B|^{1 / 2}\right)$ and $\mathfrak{b}[x, x] \leq 0$ for all $x \in \mathfrak{D}_{-}$. We then have $\mathfrak{D}_{-}=\operatorname{Ran} P_{-}$if $A$ is bounded from below and $\mathfrak{D}_{+}=\operatorname{Ran} P_{+}$if $A$ is bounded from above. Hence, item (ii) or (i), respectively, in Lemma 5.1 with $\mathcal{C}=\mathfrak{D}_{+} \oplus \mathfrak{D}_{-}$is automatically satisfied. In any case, we have by Lemma 5.1 that $I_{\operatorname{Ran} P_{+}}+X^{*} X$ maps $\mathfrak{D}_{+}$into $\mathfrak{D}_{+}$, which by identity (5.4) means that $\operatorname{Ran}\left(P_{+} Q_{+} \mid \mathfrak{D}_{+}\right) \supset \mathfrak{D}_{+}$. The representation (1.3) now follows from Proposition 3.1 (a) and Remark 3.5(1). If even $\operatorname{Dom}(A)=\operatorname{Dom}(B)$ and $\langle x, B x\rangle \leq 0$ for all $x \in \mathcal{D}_{-}$, we use the same reasoning as above with $\mathfrak{D}_{+}$and $\mathfrak{D}_{-}$replaced by $\mathcal{D}_{+}$ and $\mathcal{D}_{-}$, respectively, and obtain representation (1.4) from Proposition 3.1 (b) and Remark 3.5(1). The representation (1.3) is then still valid by Lemma 3.4 and the first part of the proof.

While certain conditions for Proposition 3.1 and Lemma 3.4 are part of the hypotheses of Theorems 1.2 and 1.3, in the situations of Theorems 1.4 and 1.5 these need to be verified explicitly from the specific hypotheses at hand. Here, we rely on previous considerations on block diagonalizations for block operator matrices and forms. In case of Theorem 1.4, the crucial ingredient is presented in the following result, extracted from [24]. An earlier result in this direction is commented on in Remark 5.4(2) below.

Proposition 5.3 (see [24,Theorem 6.1]) In the situation of Theorem 1.4 one has inequality 
$\left\|P_{+}-Q_{+}\right\| \leq \sqrt{2} / 2<1$, and the operator identity

$$
(I-Y)(A+V)(I-Y)^{-1}=A-Y V
$$

holds with $Y$ as in (5.5).

Proof Set $V_{0}:=\left.V\right|_{\operatorname{Dom}(A)}$, so that we have $B=A+V=A+V_{0}$ as well as $A-Y V=A-Y V_{0}$. Clearly, the hypotheses on $V$ ensure that $V_{0}$ is $A$-bounded with $A$-bound $b_{*}<1$ and off-diagonal with respect to the decomposition $\operatorname{Ran} P_{+} \oplus \operatorname{Ran} P_{-}$. By [24,Lemma 6.3] we now have

$$
\operatorname{Ker}\left(A+V_{0}\right) \subset \operatorname{Ker} A \subset \operatorname{Ran} P_{-} .
$$

In light of (5.2), the claim therefore is just an instance of [24,Theorem 6.1].

Remark 5.4 (1) Let $A_{ \pm}:=\left.A\right|_{\operatorname{Ran} P_{ \pm}}$be the parts of $A$ associated with the subspaces Ran $P_{ \pm}$, and write

$$
\left.V\right|_{\operatorname{Dom}(A)}=\left(\begin{array}{cc}
0 & W \\
W^{*} & 0
\end{array}\right),
$$

where $W: \operatorname{Ran} P_{-} \supset \mathcal{D}_{-} \rightarrow \operatorname{Ran} P_{+}$is given by $W x:=P_{+} V x, x \in \mathcal{D}_{-}$. Then,

$$
A-Y V=\left(\begin{array}{cc}
A_{+}-X^{*} W^{*} & 0 \\
0 & A_{-}+X W
\end{array}\right)
$$

In this sense, identity (5.7) can be viewed as a block diagonalization of the operator $A+V$. For a more detailed discussion of block diagonalizations and operator Riccati equations in the operator setting, the reader is referred to [24] and the references cited therein.

(2) In the particular case where 0 belongs to the resolvent set of $A$, the conclusion of Proposition 5.3 can be inferred also from [35, Theorems 2.7.21 and 2.8.5].

Proof of Theorem 1.4 For $x \in \mathcal{D}_{-}$, we have

$$
\langle x, V x\rangle=\left\langle P_{-} x, V P_{-} x\right\rangle=\left\langle x, P_{-} V P_{-} x\right\rangle=0
$$

and, thus,

$$
\langle x,(A+V) x\rangle=\langle x, A x\rangle \leq 0
$$

Moreover, by Proposition 5.3 the inequality $\left\|P_{+}-Q_{+}\right\|<1$ is satisfied. Let $Y$ be as in (5.5). Since $\operatorname{Dom}(A+V)=\operatorname{Dom}(A)=\operatorname{Dom}(A-Y V)$, it then follows from identity (5.7) that $I-Y$ maps $\mathcal{C}:=\operatorname{Dom}(A)=\mathcal{D}_{+} \oplus \mathcal{D}_{-}$into itself. In turn, Lemma 5.1 implies that $I_{\operatorname{Ran} P_{+}}+X^{*} X$ maps $\mathcal{D}_{+}$into itself, which by identity (5.4) means that $\operatorname{Ran}\left(P_{+} Q_{+} \mid \mathcal{D}_{+}\right) \supset \mathcal{D}_{+}$. The claim now follows from Proposition 3.1, Lemma 3.4, and Remark $3.5(1)$. 
To the best of the author's knowledge, no direct analogue of Proposition 5.3 is known so far in the setting of form rather than operator perturbations. Although the inequality $\left\|P_{+}-Q_{+}\right\| \leq \sqrt{2} / 2$ can be established here as well under fairly reasonable assumptions, see [14,Theorem 3.3], the mapping properties of the operators $I \pm Y$ connected with a corresponding diagonalization related to (5.7) are much harder to verify. The situation is even more subtle there since also the domain equality $\operatorname{Dom}\left(|A|^{1 / 2}\right)=\operatorname{Dom}\left(|B|^{1 / 2}\right)$ needs careful treatment. The latter is conjectured to hold in a general off-diagonal form perturbation framework [13, Remark 2.7]. Some characterizations have been discussed in [30,Theorem 3.8], but they all are hard to verify in a general abstract setting. A compromise in this direction is to require that the form $\mathfrak{b}$ is semibounded, see [30,Lemma 3.9] and [14,Lemma 2.7], which forces the diagonal form $\mathfrak{a}$ to be semibounded as well, see below. As in the situation of Theorem 1.3 above, this simplifies matters immensely:

Proof of Theorem 1.5 For $x \in \mathfrak{D}_{-}=\operatorname{Ran} P_{-} \cap \operatorname{Dom}[\mathfrak{a}]$ we have

$$
\mathfrak{v}\left[P_{-} x, P_{-} x\right]=0
$$

and, thus,

$$
\mathfrak{b}[x, x]=\mathfrak{a}[x, x] \leq 0 .
$$

In the same way, we see that $\mathfrak{b}[x, x]=\mathfrak{a}[x, x]$ for $x \in \mathfrak{D}_{+}$, which by the identity $\mathfrak{a}[x, x]=\mathfrak{a}\left[P_{+} x, P_{+} x\right]+\mathfrak{a}\left[P_{-} x, P_{-} x\right]$ for all $x \in \operatorname{Dom}[\mathfrak{a}]$ implies that along with $\mathfrak{b}$ also the form $\mathfrak{a}$ is semibounded; cf. the proof of [14,Lemma 2.7]. Since also $\operatorname{Dom}\left(|B|^{1 / 2}\right)=\operatorname{Dom}[\mathfrak{b}]=\operatorname{Dom}[\mathfrak{a}]=\operatorname{Dom}\left(|A|^{1 / 2}\right)$ by hypothesis and in view of Theorem 1.3 , it only remains to show that $\left\|P_{+}-Q_{+}\right\|<1$.

To this end, we first show that $\mathfrak{b}$ is a semibounded saddle-point form in the sense of [14,Section 2]: Let $m \in \mathbb{R}$ be the lower (resp. upper) bound of $\mathfrak{a}$. We then have

$$
|(\mathfrak{a}-m)[x, x]|=\left\||A-m|^{1 / 2} x\right\|^{2} \leq\left\||A-m|^{1 / 2}\left(|A|^{1 / 2}+I\right)^{-1}\right\|\left\|\left(|A|^{1 / 2}+I\right) x\right\|^{2}
$$

for all $x \in \operatorname{Dom}[\mathfrak{a}]$, where $|A-m|^{1 / 2}\left(|A|^{1 / 2}+I\right)^{-1}$ is closed and everywhere defined, hence bounded by the closed graph theorem. From this and the hypothesis on $\mathfrak{v}$ we see that

$$
|\mathfrak{v}[x, x]| \leq \beta\left(\left\||A|^{1 / 2} x\right\|^{2}+\|x\|^{2}\right) \quad \text { for all } x \in \operatorname{Dom}[\mathfrak{a}]
$$

with some $\beta \geq 0$, which means that $\mathfrak{b}=\mathfrak{a}+\mathfrak{v}=\mathfrak{a}+\mathfrak{v}_{0}$ with $\mathfrak{v}_{0}:=\left.\mathfrak{v}\right|_{\operatorname{Dom}[\mathfrak{a}]}$ is indeed a semibounded saddle-point form.

It now follows from [30,Theorem 2.13] that

Ker $B \subset \operatorname{Ker} A \subset \operatorname{Ran} P_{-}$.

In turn, [14, Theorem 3.3] and (5.2) give $\left\|P_{+}-Q_{+}\right\| \leq \sqrt{2} / 2<1$, which completes the proof. 
Acknowledgements The author is grateful to Ivan Veselić, Matthias Täufer, Stephan Schmitz, and Ivica Nakić for fruitful and inspiring discussions. He is especially indebted to Stephan Schmitz for also commenting on an earlier version of this manuscript. Finally, he thanks the anonymous referees for pointing out several references and for further comments that helped to improve the presentation of the material.

Funding Open Access funding enabled and organized by Projekt DEAL.

Data availability Data sharing not applicable to this article as no datasets were generated or analysed during the current study.

Open Access This article is licensed under a Creative Commons Attribution 4.0 International License, which permits use, sharing, adaptation, distribution and reproduction in any medium or format, as long as you give appropriate credit to the original author(s) and the source, provide a link to the Creative Commons licence, and indicate if changes were made. The images or other third party material in this article are included in the article's Creative Commons licence, unless indicated otherwise in a credit line to the material. If material is not included in the article's Creative Commons licence and your intended use is not permitted by statutory regulation or exceeds the permitted use, you will need to obtain permission directly from the copyright holder. To view a copy of this licence, visit http://creativecommons.org/licenses/by/4.0/.

\section{Appendix A: Proof of Proposition 3.1}

The following abstract minimax principle is extracted from [11, Theorem 1] and its proof follows the one in [11] almost literally. However, the result below is formulated in such a way that the operator and form settings can be handled simultaneously. To this end, we use a suitable subset $\mathcal{C}$ of $\operatorname{Dom}\left(|B|^{1 / 2}\right)$ satisfying $\operatorname{Dom}(B) \subset \mathcal{C}$. Part (a) of Proposition 3.1 then agrees with the choice $\mathcal{C}=\operatorname{Dom}\left(|B|^{1 / 2}\right)$, whereas Part (b) of Proposition 3.1 corresponds to $\mathcal{C}=\operatorname{Dom}(B)$.

Proposition A.1 (cf. [11,Theorem 1]) Assume Hypothesis 1.1. Moreover, let $\mathcal{C}$ be a subspace satisfying $\operatorname{Dom}(B) \subset \mathcal{C} \subset \operatorname{Dom}\left(|B|^{1 / 2}\right)$ such that $P_{+}$maps $\mathcal{C}$ into $\operatorname{Dom}\left(|B|^{1 / 2}\right)$. Define the subspaces $\mathcal{C}_{ \pm}:=\operatorname{Ran}\left(P_{ \pm} \mid \mathcal{C}\right) \subset \operatorname{Dom}\left(|B|^{1 / 2}\right)$, and suppose that $\mathfrak{b}[x, x] \leq 0$ for all $x \in \mathcal{C}_{-}$.

(a) For all $k \in \mathbb{N}$ with $k \leq \operatorname{dim} \operatorname{Ran} Q_{+}$we have $k \leq \operatorname{dim} \operatorname{Ran} P_{+}$and

$$
\inf _{\substack{\mathfrak{M}_{+} \subset \mathcal{C}_{+} \\ \operatorname{dim} \mathfrak{M}_{+}=k}} \sup _{\substack{x \in \mathfrak{M}_{+} \oplus \mathcal{C}_{-} \\\|x\|=1}} \mathfrak{b}[x, x] \leq \lambda_{k}\left(\left.B\right|_{\operatorname{Ran} Q_{+}}\right)
$$

(b) If, in addition, $\operatorname{Ran}\left(P_{+} Q_{+} \mid \mathcal{C}_{+}\right) \supset \mathcal{C}_{+}$and $\operatorname{Ran}\left(Q_{+} P_{+} \mid \mathcal{C}\right) \subset \mathcal{C}$, then for all $k \in \mathbb{N}$ with $k \leq \operatorname{dim} \operatorname{Ran} P_{+}$we have $k \leq \operatorname{dim} \operatorname{Ran} Q_{+}$and

$$
\inf _{\substack{\mathfrak{M}_{+} \subset \mathcal{C}_{+} \\ \operatorname{dim} \mathfrak{M}_{+}=k}} \sup _{\substack{x \in \mathfrak{M}_{+} \oplus \mathcal{C}_{-} \\\|x\|=1}} \mathfrak{b}[x, x]=\lambda_{k}\left(\left.B\right|_{\operatorname{Ran} Q_{+}}\right)
$$

Proof We first show that the restriction

$$
P_{+} \mid \mathcal{C} \cap \operatorname{Ran} Q_{+}: \mathcal{C} \cap \operatorname{Ran} Q_{+} \rightarrow \mathcal{C}_{+}
$$


is injective. Indeed, assume to the contrary that $P_{+} x=0$ for some non-zero $x \in$ $\mathcal{C} \cap \operatorname{Ran} Q_{+}$. Then, on the one hand we have $\mathfrak{b}[x, x]>0$, and on the other hand $x \in \operatorname{Ran} P_{-}$, that is, $x=P_{-} x \in \mathcal{C}_{-}$. The latter gives $\mathfrak{b}[x, x] \leq 0$ by hypothesis, a contradiction.

(a). Let $k \in \mathbb{N}$ with $k \leq \operatorname{dim} \operatorname{Ran} Q_{+}$. Let $\varepsilon>0$ be arbitrary, and abbreviate $\lambda_{k}=\lambda_{k}\left(\left.B\right|_{\operatorname{Ran} Q_{+}}\right)$. Consider the subspace

$$
\mathfrak{M}:=\operatorname{Ran} \mathrm{E}_{B}\left(\left(0, \lambda_{k}+\varepsilon\right)\right) \subset \operatorname{Dom}(B) \cap \operatorname{Ran} Q_{+} \subset \mathcal{C} \cap \operatorname{Ran} Q_{+},
$$

and denote by $P_{\mathfrak{M}}$ the orthogonal projection onto $\mathfrak{M}$. We clearly have $\operatorname{dim} \mathfrak{M} \geq k$. Choose any subspace $\mathfrak{N} \subset \mathfrak{M}$ with $\operatorname{dim} \mathfrak{N}=k$. The injectivity of (A.1) then gives $\operatorname{dim} \operatorname{Ran} P_{+} \geq\left.\operatorname{dim} \operatorname{Ran} P_{+}\right|_{\mathfrak{N}}=\operatorname{dim} \mathfrak{N}=k$.

Let $x=P_{+} u \oplus w,\|x\|=1$, with $u \in \mathfrak{N}$ and $w \in \mathcal{C}_{-}$. Then, $x=u+v$ with $v=w-P_{-} u \in \mathcal{C}_{-}$. Moreover, $\mathfrak{M}$ is reducing for $B$, that is, $P_{\mathfrak{M}}$ commutes with $B$ and, hence, also with $|B|^{1 / 2}$ and $\operatorname{sign}(B)$, see, e.g., [37,Satz 8.23]. With $P_{\mathfrak{M}} v \in \mathfrak{M}$ we therefore have

$$
\mathfrak{b}\left[v, P_{\mathfrak{M}} v\right]=\mathfrak{b}\left[P_{\mathfrak{M}} v, v\right]=\mathfrak{b}\left[P_{\mathfrak{M}} v, P_{\mathfrak{M}} v\right] \geq 0
$$

Set $x_{1}:=u+P_{\mathfrak{M}} v \in \mathfrak{M}$ and $x_{2}:=v-P_{\mathfrak{M}} v \in\left(\mathcal{C}_{-}+\mathfrak{M}\right) \cap \mathfrak{M}^{\perp}$. From (A.2) it follows that

$$
\mathfrak{b}\left[x_{2}, x_{2}\right]=\mathfrak{b}[v, v]-\mathfrak{b}\left[P_{\mathfrak{M}} v, P_{\mathfrak{M}} v\right] \leq \mathfrak{b}[v, v] \leq 0
$$

In turn, taking into account that $x=u+v=x_{1} \oplus x_{2}$ and that $\mathfrak{M}$ and $\mathfrak{M}^{\perp}$ are reducing for $B$, we obtain

$$
\mathfrak{b}[x, x]=\mathfrak{b}\left[x_{1}, x_{1}\right]+\mathfrak{b}\left[x_{2}, x_{2}\right] \leq \mathfrak{b}\left[x_{1}, x_{1}\right]<\left(\lambda_{k}+\varepsilon\right)\left\|x_{1}\right\|^{2} \leq \lambda_{k}+\varepsilon
$$

In light of $\left.\operatorname{dim} \operatorname{Ran} P_{+}\right|_{\mathfrak{N}}=k$ as observed above, we thus conclude that

$$
\inf _{\substack{\mathfrak{M}_{+} \subset \mathcal{C}_{+} \\ \operatorname{dim} \mathfrak{M}_{+}=k}} \sup _{\substack{x \in \mathfrak{M}_{+} \oplus \mathcal{C}_{-} \\\|x\|=1}} \mathfrak{b}[x, x] \leq \sup _{\substack{x \in \operatorname{Ran} P_{+} \mid \mathfrak{N} \oplus \mathcal{C}_{-} \\\|x\|=1}} \mathfrak{b}[x, x] \leq \lambda_{k}+\varepsilon
$$

Since $\varepsilon$ was chosen arbitrarily, this proves (a).

(b). The additional assumptions guarantee that the restriction (A.1) is also surjective, hence bijective. Let $k \in \mathbb{N}$ with $k \leq \operatorname{dim} \operatorname{Ran} P_{+}$, and let $\mathfrak{M}_{+}$be any subspace of $\mathcal{C}_{+}$ with $\operatorname{dim} \mathfrak{M}_{+}=k$; note that $\mathcal{C}_{+}$is dense in $\operatorname{Ran} P_{+}$, so that such a subspace indeed exists. By bijectivity of (A.1), there is a subspace $\mathfrak{M} \subset \mathcal{C} \cap \operatorname{Ran} Q_{+}$with $\operatorname{dim} \mathfrak{M}=k$ and $\mathfrak{M}_{+}=\left.\operatorname{Ran} P_{+}\right|_{\mathfrak{M}}$. In particular, we have $k \leq \operatorname{dim} \operatorname{Ran} Q_{+}$. Since $\mathfrak{M} \subset \mathcal{C}$, we have $\mathfrak{M} \subset \mathfrak{M}_{+} \oplus \mathcal{C}_{-}$and, therefore,

$$
\sup _{\substack{x \in \mathfrak{M}_{+} \oplus \mathcal{C}_{-} \\\|x\|=1}} \mathfrak{b}[x, x] \geq \sup _{\substack{x \in \mathfrak{M} \\\|x\|=1}} \mathfrak{b}[x, x] \geq \inf _{\substack{\mathfrak{N} \subset \mathcal{C} \cap \operatorname{Ran} Q_{+} \\ \operatorname{dim} \mathfrak{N}=k}} \sup _{\substack{x \in \mathfrak{N} \\\|x\|=1}} \mathfrak{b}[x, x] \geq \lambda_{k}\left(\left.B\right|_{\operatorname{Ran} Q_{+}}\right)
$$


Since $\mathfrak{M}_{+}$was chosen arbitrarily, together with part (a) this proves (b) and, thus, completes the proof.

Remark $A_{.2}$ (1) If $\mathcal{C}$ is invariant for $P_{+}$, then the subspaces $\mathcal{C}_{ \pm}$provide a splitting of $\mathcal{C}$ as $\mathcal{C}=\mathcal{C}_{+} \oplus \mathcal{C}_{-}$. Moreover, if $\mathcal{C}$ is invariant also for $Q_{+}$, then the additional assumption $\operatorname{Ran}\left(\left.Q_{+} P_{+}\right|_{\mathcal{C}}\right) \subset \mathcal{C}$ in part (b) of Proposition A.1 is automatically satisfied. This is the situation encountered in Proposition 3.1.

(2) The proof of part (b) of Proposition A.1 actually only requires that the restriction (A.1) is also surjective. This surjectivity follows from the inclusion $\operatorname{Ran}\left(P_{+} Q_{+} \mid \mathcal{C}_{+}\right) \supset \mathcal{C}_{+}$a priori only if $\mathcal{C}$ is invariant for $Q_{+} P_{+}$since only then $\operatorname{Ran}\left(\left.Q_{+}\right|_{\mathcal{C}_{+}}\right) \subset \mathcal{C} \cap \operatorname{Ran} Q_{+}$.

\section{Appendix B: Heinz Inequality}

In this appendix we discuss some consequences of the well-known Heinz inequality. These consequences or particular cases thereof are used at various spots of the main part of the paper, but they may also be of independent interest. They are probably folklore, but in lack of a suitable reference they are nevertheless presented here in full detail.

Throughout this appendix, we denote the norm associated with the inner product of a Hilbert space $\mathcal{H}$ by $\|\cdot\|_{\mathcal{H}}$.

The following variant of the Heinz inequality is taken from [20].

Proposition B.1 ( [20,Theorem I.7.1]) Let $\Lambda_{1}$ and $\Lambda_{2}$ be strictly positive self-adjoint operators on Hilbert spaces $\mathcal{H}_{1}$ and $\mathcal{H}_{2}$, respectively. Moreover, let $S: \mathcal{H}_{1} \rightarrow \mathcal{H}_{2}$ be a bounded operator mapping $\operatorname{Dom}\left(\Lambda_{1}\right)$ into $\operatorname{Dom}\left(\Lambda_{2}\right)$, and suppose that there is a constant $C \geq 0$ such that

$$
\left\|\Lambda_{2} S x\right\|_{\mathcal{H}_{2}} \leq C\left\|\Lambda_{1} x\right\|_{\mathcal{H}_{1}} \quad \text { for all } x \in \operatorname{Dom}\left(\Lambda_{1}\right)
$$

Then, for all $v \in[0,1]$, the operator $S$ maps $\operatorname{Dom}\left(\Lambda_{1}^{v}\right)$ into $\operatorname{Dom}\left(\Lambda_{2}^{v}\right)$, and for all $x \in \operatorname{Dom}\left(\Lambda_{1}^{v}\right)$ one has

$$
\left\|\Lambda_{2}^{v} S x\right\|_{\mathcal{H}_{2}} \leq C^{v}\|S\|_{\mathcal{H}_{1} \rightarrow \mathcal{H}_{2}}^{1-v}\left\|\Lambda_{1}^{v} x\right\|_{\mathcal{H}_{1}}
$$

The above result admits the following extension to closed densely defined operators between Hilbert spaces. For a generalization of Proposition B.1 to maximal accretive operators, see [16].

Proposition B.2 Let $\mathcal{H}_{1}, \mathcal{H}_{2}, \mathcal{K}_{1}$, and $\mathcal{K}_{2}$ be Hilbert spaces, and let $\Lambda_{1}: \mathcal{H}_{1} \supset$ $\operatorname{Dom}\left(\Lambda_{1}\right) \rightarrow \mathcal{K}_{1}$ and $\Lambda_{2}: \mathcal{H}_{2} \supset \operatorname{Dom}\left(\Lambda_{2}\right) \rightarrow \mathcal{K}_{2}$ be closed densely defined operators. Moreover, let $S: \mathcal{H}_{1} \rightarrow \mathcal{H}_{2}$ be a bounded operator mapping $\operatorname{Dom}\left(\Lambda_{1}\right)$ into $\operatorname{Dom}\left(\Lambda_{2}\right)$, and suppose that there is a constant $C \geq 0$ such that

$$
\left\|\Lambda_{2} S x\right\|_{\mathcal{K}_{2}} \leq C\left\|\Lambda_{1} x\right\|_{\mathcal{K}_{1}} \text { for all } x \in \operatorname{Dom}\left(\Lambda_{1}\right)
$$

Then, for all $v \in[0,1]$, the operator $S$ maps $\operatorname{Dom}\left(\left|\Lambda_{1}\right|^{v}\right)$ into $\operatorname{Dom}\left(\left|\Lambda_{2}\right|^{v}\right)$. 
Proof Recall that $\left\|\Lambda_{j} y\right\|_{\mathcal{K}_{j}}=\left\|\left|\Lambda_{j}\right| y\right\|_{\mathcal{H}_{j}}$ for all $y \in \operatorname{Dom}\left(\Lambda_{j}\right)=\operatorname{Dom}\left(\left|\Lambda_{j}\right|\right)$, $j=1,2$. Moreover, the operator $S$ maps domain $\operatorname{Dom}\left(\left|\Lambda_{1}\right|+I_{\mathcal{H}_{1}}\right)=\operatorname{Dom}\left(\Lambda_{1}\right)$ into $\operatorname{Dom}\left(\left|\Lambda_{2}\right|+I_{\mathcal{H}_{2}}\right)=\operatorname{Dom}\left(\Lambda_{2}\right)$ by hypothesis. We estimate

$$
\begin{aligned}
\left\|\left(\left|\Lambda_{2}\right|+I_{\mathcal{H}_{2}}\right) S x\right\|_{\mathcal{H}_{2}} & \leq\left\|\Lambda_{2} S x\right\|_{\mathcal{K}_{2}}+\|S x\|_{\mathcal{H}_{2}} \leq C\left\|\Lambda_{1} x\right\|_{\mathcal{K}_{1}}+\|S x\|_{\mathcal{H}_{2}} \\
& \leq \widetilde{C}\left\|\left(\left|\Lambda_{1}\right|+I_{\mathcal{H}_{1}}\right) x\right\|_{\mathcal{H}_{1}}
\end{aligned}
$$

for all $x \in \operatorname{Dom}\left(\Lambda_{1}\right)$ with

$$
\widetilde{C}:=C\left\|\Lambda_{1}\left(\left|\Lambda_{1}\right|+I_{\mathcal{H}_{1}}\right)^{-1}\right\|_{\mathcal{H}_{1} \rightarrow \mathcal{K}_{1}}+\left\|S\left(\left|\Lambda_{1}\right|+I_{\mathcal{H}_{1}}\right)^{-1}\right\|_{\mathcal{H}_{1} \rightarrow \mathcal{H}_{2}}
$$

Here, we have taken into account that $\Lambda_{1}\left(\left|\Lambda_{1}\right|+I_{\mathcal{H}_{1}}\right)^{-1}$ is a closed and everywhere defined operator from $\mathcal{H}_{1}$ to $\mathcal{K}_{1}$, hence bounded by the closed graph theorem.

Applying Proposition B.1 now yields that $S$ maps $\operatorname{Dom}\left(\left(\left|\Lambda_{1}\right|+I_{\mathcal{H}_{1}}\right)^{v}\right)$ into $\operatorname{Dom}\left(\left(\left|\Lambda_{2}\right|+I_{\mathcal{H}_{2}}\right)^{v}\right)$ for all $v \in[0,1]$. It remains to observe that by functional calculus one has $\operatorname{Dom}\left(\left(\left|\Lambda_{j}\right|+I_{\mathcal{H}_{j}}\right)^{v}\right)=\operatorname{Dom}\left(\left|\Lambda_{j}\right|^{v}\right)$ for $j \in\{1,2\}$, which completes the proof.

We now obtain several easy corollaries.

Corollary B.3 (cf. [30,Corollary 3.3]) Let $\mathcal{H}, \mathcal{K}_{1}$, and $\mathcal{K}_{2}$ be Hilbert spaces, and let $\Lambda_{1}: \mathcal{H} \supset \operatorname{Dom}\left(\Lambda_{1}\right) \rightarrow \mathcal{K}_{1}$ and $\Lambda_{2}: \mathcal{H} \supset \operatorname{Dom}\left(\Lambda_{2}\right) \rightarrow \mathcal{K}_{2}$ be closed densely defined operators.

If $\operatorname{Dom}\left(\Lambda_{1}\right) \subset \operatorname{Dom}\left(\Lambda_{2}\right)$, then $\operatorname{Dom}\left(\left|\Lambda_{1}\right|^{v}\right) \subset \operatorname{Dom}\left(\left|\Lambda_{2}\right|^{v}\right)$ for all $v \in[0,1]$. Moreover, if $\operatorname{Dom}\left(\Lambda_{1}\right)=\operatorname{Dom}\left(\Lambda_{2}\right)$, then also $\operatorname{Dom}\left(\left|\Lambda_{1}\right|^{v}\right)=\operatorname{Dom}\left(\left|\Lambda_{2}\right|^{v}\right)$ for all $v \in[0,1]$.

Proof Suppose that $\operatorname{Dom}\left(\Lambda_{1}\right) \subset \operatorname{Dom}\left(\Lambda_{2}\right)$. Since $\operatorname{Dom}\left(\left|\Lambda_{1}\right|\right)=\operatorname{Dom}\left(\Lambda_{1}\right)$, we have as in the proof of the preceding proposition that $\Lambda_{2}\left(\left|\Lambda_{1}\right|+I_{\mathcal{H}}\right)^{-1}$ is a closed everywhere defined, hence bounded, operator from $\mathcal{H}$ to $\mathcal{K}_{2}$. Thus,

$$
\left\|\Lambda_{2} x\right\|_{\mathcal{K}_{2}} \leq\left\|\Lambda_{2}\left(\left|\Lambda_{1}\right|+I_{\mathcal{H}}\right)^{-1}\right\|_{\mathcal{H} \rightarrow \mathcal{K}_{2}} \cdot\left\|\left(\left|\Lambda_{1}\right|+I_{\mathcal{H}}\right) x\right\|_{\mathcal{H}}
$$

for all $x \in \operatorname{Dom}\left(\Lambda_{1}\right)$, and applying Proposition B.2 with $S=I_{\mathcal{H}}$ yields that

$$
\operatorname{Dom}\left(\left|\Lambda_{1}\right|^{v}\right)=\operatorname{Dom}\left(\left(\left|\Lambda_{1}\right|+I_{\mathcal{H}}\right)^{v}\right) \subset \operatorname{Dom}\left(\left|\Lambda_{2}\right|^{v}\right)
$$

If also $\operatorname{Dom}\left(\Lambda_{1}\right) \supset \operatorname{Dom}\left(\Lambda_{2}\right)$, the above with switched roles of $\Lambda_{1}$ and $\Lambda_{2}$ yields that also $\operatorname{Dom}\left(\left|\Lambda_{2}\right|^{v}\right) \subset \operatorname{Dom}\left(\left|\Lambda_{1}\right|^{v}\right)$, which completes the proof.

Remark B.4 In the particular case of $v=1 / 2$, Corollary B.3 can alternatively be proved with classical considerations regarding operator and form boundedness:

If $\operatorname{Dom}\left(\Lambda_{1}\right) \subset \operatorname{Dom}\left(\Lambda_{2}\right)$, then also $\operatorname{Dom}\left(\left|\Lambda_{1}\right|\right) \subset \operatorname{Dom}\left(\left|\Lambda_{2}\right|\right)$, so that $\left|\Lambda_{2}\right|$ is relatively operator bounded with respect to $\left|\Lambda_{1}\right|$, see, e.g., [17,Remark IV.1.5]. In turn, by [17, Theorem VI.1.38], $\left|\Lambda_{2}\right|$ is also form bounded with respect to $\left|\Lambda_{1}\right|$, which extends to the closure of the forms. The latter includes that $\operatorname{Dom}\left(\left|\Lambda_{1}\right|^{1 / 2}\right) \subset \operatorname{Dom}\left(\left|\Lambda_{2}\right|^{1 / 2}\right)$. 
Corollary B.5 Let $\Lambda_{1}$ and $\Lambda_{2}$ be as in Proposition B.2, and suppose that operator $S: \mathcal{H}_{1} \rightarrow \mathcal{H}_{2}$ is bounded and bijective with $\operatorname{Ran}\left(\left.S\right|_{\operatorname{Dom}\left(\Lambda_{1}\right)}\right)=\operatorname{Dom}\left(\Lambda_{2}\right)$. Then, one has $\operatorname{Ran}\left(\left.S\right|_{\operatorname{Dom}\left(\left|\Lambda_{1}\right|^{\nu}\right)}\right)=\operatorname{Dom}\left(\left|\Lambda_{2}\right|^{\nu}\right)$ for all $v \in[0,1]$.

Proof Consider the closed densely defined operator $\Lambda_{3}:=\Lambda_{1} S^{-1}$ with domain $\operatorname{Dom}\left(\Lambda_{3}\right)=\operatorname{Ran}\left(\left.S\right|_{\operatorname{Dom}\left(\Lambda_{1}\right)}\right)=\operatorname{Dom}\left(\Lambda_{2}\right)$. By definition, $S$ maps $\operatorname{Dom}\left(\Lambda_{1}\right)$ onto $\operatorname{Dom}\left(\Lambda_{3}\right)$. Moreover, we have $\Lambda_{3} S x=\Lambda_{1} x$ and, in particular,

$$
\left\|\Lambda_{3} S x\right\|_{\mathcal{K}_{1}}=\left\|\Lambda_{1} x\right\|_{\mathcal{K}_{1}}
$$

for all $x \in \operatorname{Dom}\left(\Lambda_{1}\right)$. Proposition B.2 now implies that $S$ maps $\operatorname{Dom}\left(\left|\Lambda_{1}\right|^{v}\right)$ into $\operatorname{Dom}\left(\left|\Lambda_{3}\right|^{v}\right)$. Since $\operatorname{Dom}\left(\left|\Lambda_{3}\right|^{v}\right)=\operatorname{Dom}\left(\left|\Lambda_{2}\right|^{v}\right)$ for all $v \in[0,1]$ in light of Corollary B.3, this proves the inclusion $\operatorname{Ran}\left(\left.S\right|_{\operatorname{Dom}\left(\left|\Lambda_{1}\right|^{\nu}\right)}\right) \subset \operatorname{Dom}\left(\left|\Lambda_{2}\right|^{\nu}\right)$.

Since $S$ is bijective and $S^{-1}$ maps $\operatorname{Dom}\left(\Lambda_{2}\right)$ onto $\operatorname{Dom}\left(\Lambda_{1}\right)$ by hypothesis, one verifies in an analogous way that $S^{-1} \operatorname{maps} \operatorname{Dom}\left(\left|\Lambda_{2}\right|^{v}\right)$ into $\operatorname{Dom}\left(\left|\Lambda_{1}\right|^{v}\right)$. This shows the converse inclusion and, hence, completes the proof.

The last corollary discussed here is related to the question whether an operator sum represents the sum of the corresponding forms. Part (b) of this corollary can in some sense also be regarded as an extension of [29,Lemma 2.2.7] to not necessarily off-diagonal perturbations.

Corollary B.6 (cf. [29,Lemma 2.2.7]) Let $\Lambda$ be a self-adjoint operator on a Hilbert space with inner product $\langle\cdot, \cdot\rangle$, and let $K$ be an operator on the same Hilbert space.

(a) If $K$ is symmetric with $\operatorname{Dom}(K) \supset \operatorname{Dom}\left(|\Lambda|^{1 / 2}\right)$, then the operator sum $\Lambda+K$ defines a self-adjoint operator with

$$
\begin{aligned}
\left\langle|\Lambda+K|^{1 / 2} x, \operatorname{sign}(\Lambda+K)|\Lambda+K|^{1 / 2} y\right\rangle= & \left\langle|\Lambda|^{1 / 2} x, \operatorname{sign}(\Lambda)|\Lambda|^{1 / 2} y\right\rangle \\
& +\langle x, K y\rangle
\end{aligned}
$$

for all $x, y \in \operatorname{Dom}\left(|\Lambda|^{1 / 2}\right)=\operatorname{Dom}\left(|\Lambda+K|^{1 / 2}\right)$.

(b) If $K$ is self-adjoint with $\operatorname{Dom}(K) \supset \operatorname{Dom}(\Lambda)$ such that $\Lambda+K$ is self-adjoint on $\operatorname{Dom}(\Lambda+K)=\operatorname{Dom}(\Lambda)$, then

$$
\begin{array}{r}
\left\langle|\Lambda+K|^{1 / 2} x, \operatorname{sign}(\Lambda+K)|\Lambda+K|^{1 / 2} y\right\rangle \\
=\left\langle|\Lambda|^{1 / 2} x, \operatorname{sign}(\Lambda)|\Lambda|^{1 / 2} y\right\rangle+\left\langle|K|^{1 / 2} x, \operatorname{sign}(K)|K|^{1 / 2} y\right\rangle
\end{array}
$$

for all $x, y \in \operatorname{Dom}\left(|\Lambda|^{1 / 2}\right)=\operatorname{Dom}\left(|\Lambda+K|^{1 / 2}\right)$.

Proof (a). For $x, y \in \operatorname{Dom}(\Lambda)=\operatorname{Dom}(\Lambda+K)$, both sides of the claimed identity clearly agree. For general $x, y \in \operatorname{Dom}\left(|\Lambda|^{1 / 2}\right)=\operatorname{Dom}\left(|\Lambda+K|^{1 / 2}\right)$, we use that $\operatorname{Dom}(\Lambda)$ is an operator core for $|\Lambda|^{1 / 2}$ and approximate both $x$ and $y$ within $\operatorname{Dom}(\Lambda)$ with respect to the graph norm of $|\Lambda|^{1 / 2}$. In order to conclude the claim, it only remains to show that $x$ and $y$ are then also approximated with respect to the graph norms of $K$ and $|\Lambda+K|^{1 / 2}$, respectively. For this it suffices to establish that $K$ and $|\Lambda+K|^{1 / 2}$ are operator bounded with respect to $|\Lambda|^{1 / 2}$. 
That $K$ is operator bounded with respect to $|\Lambda|^{1 / 2}$ follows immediately from the inclusion $\operatorname{Dom}\left(|\Lambda|^{1 / 2}\right) \subset \operatorname{Dom}(K)$ and the fact that $K$ is closable, see, e.g., [17, Remark IV.1.5 and Section V.3.3]. Moreover, the same properties yield by Corollary 2.1.20 in [35] that $K$ is operator infinitesimal with respect to $\Lambda$. In particular, the operator sum $\Lambda+K$ is self-adjoint on $\operatorname{Dom}(\Lambda+K)=\operatorname{Dom}(\Lambda)$ by the wellknown Kato-Rellich theorem. In turn, Corollary B.3 implies that $\operatorname{Dom}\left(|\Lambda+K|^{1 / 2}\right)=$ $\operatorname{Dom}\left(|\Lambda|^{1 / 2}\right)$, so that also $|\Lambda+K|^{1 / 2}$ is operator bounded with respect to $|\Lambda|^{1 / 2}$. This completes the proof of (a).

(b). Corollary B.3 implies that $\operatorname{Dom}\left(|\Lambda+K|^{1 / 2}\right)=\operatorname{Dom}\left(|\Lambda|^{1 / 2}\right)$ and Dom $\left(|\Lambda|^{1 / 2}\right) \subset \operatorname{Dom}\left(|K|^{1 / 2}\right)$. In turn, as in part (a), both $|\Lambda+K|^{1 / 2}$ and $|K|^{1 / 2}$ are relatively bounded with respect to $|\Lambda|^{1 / 2}$. The claimed identity now follows just as in part (a) by approximation upon observing that it certainly holds for $x, y \in$ $\operatorname{Dom}(\Lambda)$.

\section{References}

1. Adamjan, V.M., Langer, H.: Spectral properties of a class of rational operator valued functions. J. Oper. Theory 33, 259-277 (1995)

2. Datta, S.N., Devaiah, G.: The minimax technique in relativistic Hartree-Fock calculations. Pramana 30, 387-405 (1988)

3. Davis, C., Kahan, W.M.: The rotation of eigenvectors by a perturbation. III. SIAM J. Numer. Anal. 7, $1-46(1970)$

4. Dolbeault, J., Esteban, M.J., Loss, M., Vega, L.: An analytical proof of Hardy-like inequalities related to the Dirac operator. J. Funct. Anal. 216, 1-21 (2004)

5. Dolbeault, J., Esteban, M.J., Séré, E.: Variational characterization for eigenvalues of Dirac operators. Calc. Var. Part. Differ. Equ. 10, 321-347 (2000)

6. Dolbeault, J., Esteban, M.J., Séré, E.: On the eigenvalues of operators with gaps. Application to Dirac Operators. J. Funct. Anal. 174, 208-226 (2000)

7. Eschwé, D., Langer, H.: Triple variational principles for eigenvalues of self-adjoint operators and operator functions. SIAM J. Math. Anal. 34, 228-238 (2002)

8. Esteban, M.J., Lewin, M., Séré, E.: Domains for Dirac-Coulomb min-max levels. Rev. Mat. Iberoam. 35, 877-924 (2019)

9. Esteban, M. J., Séré, E.: Existence and multiplicity of solutions for linear and non-linear Dirac problems. In: Partial Differential Equations and Their Applications (Toronto, ON, 1995), CRM Proc. Lecture Notes, vol. 12, Amer. Math. Soc., RI, pp. 107-118 (1997)

10. Faierman, M., Fries, R.J., Mennicken, R., Möller, M.: On the essential spectrum of the linearized Navier-Stokes operator. Integr. Equ. Oper. Theory 38, 9-27 (2000)

11. Griesemer, M., Lewis, R.T., Siedentop, H.: A minimax principle for eigenvalues in spectral gaps: Dirac operators with Coulomb potentials. Doc. Math. 4, 275-283 (1999)

12. Griesemer, M., Siedentop, H.: A minimax principle for eigenvalues in spectral gaps. J. Lond. Math. Soc. (2) 60, 490-500 (1999)

13. Grubišić, L., Kostrykin, V., Makarov, K.A., Veselić, K.: The Tan2 $\Theta$ Theorem for indefinite quadratic forms. J. Spectr. Theory 3, 83-100 (2013)

14. Grubišić, L., Kostrykin, V., Makarov, K. A., Schmitz, S., Veselić, K.: Diagonalization of indefinite saddle point forms. In: Analysis as a Tool in Mathematical Physics: in Memory of Boris Pavlov, Oper. Theory Adv. Appl., vol. 276, Birkhäuser, Basel, pp. 373-400 (2020)


dynamics. J. Spectr. Theory 9, 1431-1457 (2019)

16. Kato, T.: A generalization of the Heinz inequality. Proc. Japan Acad. 37, 305-308 (1961)

17. Kato, T.: Perturbation Theory for Linear Operators. Classics Math. Springer, Berlin (1995)

18. Kostrykin, V., Makarov, K.A., Motovilov, A.K.: Existence and uniqueness of solutions to the operator Riccati equation. A geometric approach. In: Advances in Differential Equations and Mathematical 
Physics (Birmingham, AL, 2002), Contemp. Math., vol. 327, Amer. Math. Soc., Providence, RI, pp. 181-198 (2003)

19. Kraus, M., Langer, M., Tretter, C.: Variational principles and eigenvalue estimates for unbounded block operator matrices and applications. J. Comput. Appl. Math. 171, 311-334 (2004)

20. Krĕ̌n, S.G.: Linear Differential Equations in Banach Space. Transl. Math. Monogr., vol. 29, Amer. Math. Soc., Providence, RI (1969)

21. Langer, H., Langer, M., Tretter, C.: Variational principles for eigenvalues of block operator matrices. Indiana Univ. Math. J. 51, 1427-1459 (2002)

22. Langer, M., Strauss, M.: Triple variational principles for self-adjoint operator functions. J. Funct. Anal. 270, 2019-2047 (2016)

23. Lieb, E.H., Loss, M.: Analysis, 2nd edn, Grad. Stud. Math., vol. 14, Amer. Math. Soc., Providence, RI (2001)

24. Makarov, K.A., Schmitz, S., Seelmann, A.: On invariant graph subspaces. Integr. Equ. Oper. Theory 85, 399-425 (2016)

25. Morozov, S., Müller, D.: On the minimax principle for Coulomb-Dirac operators. Math. Z. 280, 733747 (2015)

26. Motovilov, A.K., Selin, A.V.: Some sharp norm estimates in the subspace perturbation problem. Integr. Equ. Oper. Theory 56, 511-542 (2006)

27. Nakić, I., Täufer, M., Tautenhahn, M., Veselić, I.: Unique continuation and lifting of spectral band edges of Schrödinger operators on unbounded domains, with an appendix by Albrecht Seelmann. J. Spectr. Theory 10, 843-885 (2020)

28. Schimmer, L., Solovej, J.P., Tokus, S.: Friedrichs extension and min-max principle for operators with a gap. Ann. Henri Poincaré 21, 327-357 (2020)

29. Schmitz, S.: Representation theorems for indefinite quadratic forms and applications. Dissertation, Johannes Gutenberg-Universität Mainz (2014)

30. Schmitz, S.: Representation theorems for indefinite quadratic forms without spectral gap. Integr. Equ. Oper. Theory 83, 73-94 (2015)

31. Schmüdgen, K.: Unbounded Self-Adjoint Operators on Hilbert Space. Grad. Texts in Math., vol. 265. Springer, Dordrecht (2012)

32. Seelmann, A.: Perturbation theory for spectral subspaces. Dissertation, Johannes GutenbergUniversität Mainz (2014)

33. Seelmann, A.: Unifying the treatment of indefinite and semidefinite perturbations in the subspace perturbation problem. Oper. Matrices 15, 1181-1188 (2021)

34. Talman, J.D.: Minimax principle for the Dirac equation. Phys. Rev. Lett. 57, 1091-1094 (1986)

35. Tretter, C.: Spectral Theory of Block Operator Matrices and Applications. Imperial College Press, London (2008)

36. Veselić, K.: Spectral perturbation bounds for selfadjoint operators. I. Oper. Matrices 2, 307-339 (2008)

37. Weidmann, J.: Lineare Operatoren in Hilberträumen. Teil 1. Grundlagen. Mathematische Leitfäden, B.G. Teubner, Stuttgart (2000)

Publisher's Note Springer Nature remains neutral with regard to jurisdictional claims in published maps and institutional affiliations. 